Originalveröffentlichung in: Steiger, Johann Anselm u.a. (Hrsg.): Innovation durch Wissenstransfer in der Frühen Neuzeit, Amsterdam (u.a.) 2010, S. 343-384 (Chloe ; 41)

Ulrich Pfisterer

\title{
MYTHEN VON KÜNSTLERISCHER INNOVATION UND TRADITION
}

\author{
Annibale Carraccis Almosenspende des Hl. Rochus \\ und die Erneuerung der christlichen Malerei um 1600
}

\section{Abstract}

In den Diskussionen und Theorien über die Bildkünste gewinnt in den Jahrzehnten um 1600 eine zuvor in dieser Form unbekannte Idee zentrale Bedeutung: die Vorstellung, daß die bedeutendsten Künstler 'voraussetzungslose' Werke schaffen können und sollen. Innovation läßt sich nun nicht mehr nur im Rahmen von Imitatio-Lehren begreifen, sondern auch als radikaler Traditionsbruch und Neuanfang (ein Mythos, auf den sich prinzipiell auch noch die Avantgarden um 1900 beriefen). Dabei war im kunsttheoretischen Verständnishorizont der Frühen Neuzeit diese bedingungslose novità nur zu verwirklichen, wenn ein Künstler ohne Lehrer (will sagen: ohne jede Traditionsanbindung) als Autodidakt und aufgrund angeborener Begabung einen Stil der unmittelbaren und 'nicht-idealisierten' Naturnachahmung entwickelte. Am Beispiel von Annibale Carraccis 1595 vollendeter Almosenspende des Hl. Rochus für eine Bruderschaftskirche in Reggio Emilia wird exemplarisch gezeigt, welche Konsequenzen diese Vorstellungen für das künstlerische Schaffen und den Umgang mit der Bildtradition hatten. Carracci versuchte, mit diesem monumentalen Gemälde eine neue Form des christlichen Historienbildes zu etablieren.

Im Jahr 1633 mußte sich der große Gianlorenzo Bernini, dessen Werke aus heutiger Sicht allesamt als revolutionäre künstlerische Innovationen erscheinen, im Gespräch mit dem obskuren Literaten Lelio Guidiccioni belehren lassen, daß solche radikalen Neuerungen weder für die "nobili arti" noch die "scienze" erstrebenswert seien. Es ginge vielmehr um das geistreich-überraschende Aufdecken von neuen, ungewohnten Aspekten innerhalb des Tradierten:

G. L. [Bernini]: Man kann also keine Innovation tätigen, noch etwas Eigenes finden, noch irgend etwas zum von anderen Erfundenen hinzufügen? [...]. Ich hielt es [bislang eigentlich] für ehrenvoller, auf neuen Wegen zu gehen als auf der alten Straße - und diese [auch noch] zu verlieren angesichts der Menge, die auf dieser dahinwogt. L. [Guidiccioni]: Falsch! Man soll auf der alten Straße gehen, aber mit neuer Wertsetzung, indem man nicht die 
etablierte Richtung und die Menge flieht, sondern sich abhebt, das Triviale meidet, sich zur Perfektion aufschwingt. ${ }^{1}$

Guidiccionis kurzer Dialog faßt in konziser Form Diskussionen zusammen, die während der letzten fünf Jahrzehnte in Italien und darüber hinaus immer größere Bedeutung gewonnen hatten - Diskussionen über das Wesen und die Möglichkeiten von novità, von Neuerungen des Denkens und Wissens, der Technik, des Handwerks sowie der literarischen und künstlerischen Produktion im weitesten Sinne. ${ }^{2}$

Nun hat es ein Bewußtsein von 'Neuem' wohl zu allen Zeiten und wohl immer auch schon mit kontroversen Meinungen dazu gegeben: sei es in Form kleiner Fortschritte, wie es das von einigen Autoren des Mittelalters gerne benutzte Bild von den Zwergen auf den Schultern von Riesen veranschaulicht, oder aber in Form eines Renovatio-Denkens, bei dem frühere, meist antike Errungenschaften, die zeitweise verloren gegangen waren, wiederbelebt werden. ${ }^{3} \mathrm{Da}$ gerade in den Künsten das Tempo dieser Neuerungen vom 15. bis zum früheren 17. Jahrhundert eine seiner größten Beschleunigungen erfährt, ist ebenfalls leicht nachvollziehbar. Aber bei dem Dialog zwischen Bernini und Guidiccioni geht es nicht um 'Innovation' an sich, sondern der Bildhauer vertritt die viel radikalere Behauptung, es gäbe die Möglichkeit quasi voraussetzungsloser,

1 Nach der Handschrift in der Vatikanischen Bibliothek ediert von Cesare D'Onofrio: Note berniniane 1 - Un dialogo-recita di Gian Lorenzo Bernini e Lelio Guidiccioni. In: Palatino 10 (1966), S. 127-134, der originale Wortlaut der frei übersetzten Passage lautet: "G. L.: Dunque non si può innovare, né trovare di proprio, né aggiunger cosa alcuna all'inventato da gli altri? [...]. Più glorioso stimavo il caminare per nuova strada che andando per l'antica, perderla trà la moltitudine che v'ondeggia per entro. L.: Falso. Si vuol caminare per la strada vecchia, ma con nuovo valore, non fuggire il concorso, et la moltitudine, ma segnalarsi da essa, et abhorrendo la trivialità, sollevarsi al perfetto, [...]."

2 Die prinzipielle Bedeutung dieser Frage für die Kunstgeschichte hat jüngst Elizabeth Cropper: The Domenichino Affair. Novelty, Imitation, and Theft in Seventeenth-Century Rome. New Haven/London 2005 betont, allerdings nimmt sie nur eine Form von 'Neuheit', nämlich kreative Aneignung der Tradition, in den Blick.

3 Etwa David Quint: Origin and Originality in Renaissance Literature. New Haven/ London 1983; Walter Haug, Burghart Wachinger (Hrsg.): Innovation und Originalität. Tübingen 1993; François Laroque, Frank Lessay (Hrsg.): Innovation et tradition de la Renaissance aux lumières. Paris 2002; Hans-Joachim Schmidt (Hrsg.): Tradition, Innovation, Invention: Fortschrittsverweigerung und Fortschrittsbewußtsein im Mittelalter. Berlin u. a. 2005. 
die Tradition negierender künstlerischer Neuerung. Zwar lassen sich auch in diesem forcierten Sinne bereits kurz nach 1400 Äußerungen finden: So spricht etwa Leon Battista Alberti 1436 im Vorwort der Volgare-Fassung seines Malerei-Traktats von "nie gesehenen Künsten". ${ }^{4}$ Und sein Zeitgenosse, der Naturforscher und Arzt Giovanni Fontana, konzediert den Künstlern sogar eine "creatio ex nihilo". ${ }^{5}$ Ähnlich spielen in den Diskussionen zwischen antiqui und moderni über den Stellenwert antiker und zeitgenössischer Kultur die Bildkünste eine Rolle als Argument für den Fortschritt der Gegenwart - allerdings kaum in stilistischer, sondern vor allem in technischer Hinsicht, so insbesondere die Erfindung der Zentralperspektive, der Ölmalerei und der Drucktechniken (s. Abb. 1). ${ }^{6} \mathrm{Im}$ 16. Jahrhundert scheint dann etwa in Vasaris Künstler-Viten an einigen Stellen von vollkommen neuen künstlerischen Inventionen die Rede zu sein; zur gleichen Zeit propagieren zahlreiche Manieristen in ihren Werken selbst ihren Erfindungsreichtum. Entscheidend ist jedoch, daß diese theoretischen oder praktischen Äußerungen zu den Künsten, für die sich noch zahlreiche weitere Beispiele anführen ließen, zumindest für ein größeres Publikum in umfassende Renaissance- und FortschrittsKonzepte eingebunden bleiben, die im größeren Rahmen diese radikalen Neuerungen wieder relativieren: So mildert etwa Vasari selbst die

4 Leon Battista Alberti: Della Pittura. Über die Malkunst. Hrsg. von Oskar Bätschmann, Sandra Gianfreda. Darmstadt 2002, S. 62-65: “[...] se noi sanza precettori, senza essemplo alcuno, troviamo arti e scienze non udite e mai vedute". - Zu Albertis ambivalentem Bemühen um solche "res novas, inauditas" (Vorwort Momus) s. auch Alan F. Nagel: Rhetoric, Value, and Action in Alberti. In: Modern Language Notes 95 (1980), S. 39-65; Luigi Trenti: 'Nihil dictum quin prius dictum'. La fenomenologia sentenziosa di Leon Battista Alberti. In: Quaderni di Retorica e Poetica 2 (1986), S. 51-62.

5 Nach Ulrich Pfisterer: Die Kunstliteratur der italienischen Renaissance. Eine Geschichte in Quellen. Stuttgart 2002, S. 201 (Nr. 91).

6 Vgl. etwa Antonio Averlino detto il Filarete: Trattato di Architettura. Hrsg. von Anna M. Finoli, Liliana Grassi. Mailand 1972, Bd. 2, S. 657 zur Erfindung der Perspektive durch Brunelleschi; Camillo Leonardi in seinem 1502 publizierten Speculum Lapidum zu Drucktechniken (die relevante Passage auch ediert bei Eugenio Garin: Giudizi artistici di Camillo Lunardi. In: Rinascimento 2 [1951], S. 191): "unum apud modernos respicio, de quo apud antiquos nulla extat memoria, de incisoribus seu sculptoribus in argento, quae sculptura niellum appellatur [...].” Uta Bernsmeier: Die Nova Reperta des Jan van der Straet. Ein Beitrag zur Problemgeschichte der Entdeckungen und Erfindungen im 16. Jahrhundert. Diss. Hamburg 1986. 
fundamentale Wiederbelebung der Künste um 1300 durch Giotto u.a. dadurch $\mathrm{ab}$, daß seine Viten mit dem Vorläufer Cimbue, der seinerseits auf einige Griechen rekurrierte, einsetzt und dann noch mehrere Künstler in der Reihenfolge der Lebensbeschreibungen zwischen Cimabue und dessen 'Schüler' Giotto eingeschoben wurden. Und für die Manieristen läßt sich etwas pauschalisierend sagen, daß sie alle an einer Weiterführung und Übersteigerung der künstlerischen Tradition und vor allem der Kunstfiguren Michelangelos arbeiten, nicht an einer radikalen Absetzung von diesen. ${ }^{7}$ Vielleicht die besten Anwärter auf voraussetzungslose Innovation im 16. Jahrhundert finden sich bezeichnenderweise auBerhalb der europäischen Tradition in der Neuen Welt: So bestaunte man beispielsweise als etwas vollkommen Neues die Federbilder der dortigen Ureinwohner. ${ }^{8}$

Die Gewichtung dieser Vorstellungen verschiebt sich zumindest bei einigen Autoren nun maßgeblich in der zweiten Hälfte des 16. Jahrhunderts. Jetzt kann ein Genueser Schriftsteller in Anlehnung an Kolumbus die radikale Maxime ausgeben, "neue Welten" (der Dichtung) zu finden oder aber unterzugehen. ${ }^{9}$ Bücher zu den Neuerfindungen der 'Moderne' und zur eigenständigen Bedeutung der Gegenwart - explizit auch der Bildkünste - überfluten den Markt. ${ }^{10}$ Und insbesondere der

7 Vgl. etwa (je mit weiterer Lit.) Philip Sohm: Ordering history with style. Giorgio Vasari on the art of history. In: Alina A. Payne (Hrsg.): Antiquity and its interpreters. Cambridge u. a. 2000, S. 40-54; Melinda Wilcox Schlitt: "Anticamente moderna e modernamente antica". Imitation and the ideal in 16th-century Italian painting. In: International Journal of the Classical Tradition 10 (2003), S. 377-406.

8 Guido Panciroli: Rerum memorabilium iam olim deperditarum et contra recens atque ingeniose inventarum libri II. Amberg 1599-1600, Bd. 2, S. 173.

9 "Diceva ch'egli seguiva Cristofaro Colombo suo concittadino, ch'egli voleva trovar nuovo mondo, o affogar" - so Gabriello Chiabrera in seiner Autobiographie (Ders.: Delle Opere. Venedig 1757, Bd. 1).

10 Neben der um 1590 publizierten Stichserie der Nova Reperta von Stradanus etwa Panciroli (s. Anm. 9); Alessandro Tassoni: Ingegni antichi e moderni (Pensieri X). In: Ders.: Pensieri e scritti preparatori. Hrsg. von Pietro Puliatti. Modena 1986 (zuerst 1620), S. 841-934, v. a. S. 878-888 (cap. xvii 'Fabbriche antiche e moderne') und S. 893-901 (cap. xix 'Statue e pitture antiche e moderne'); Secondo Lancellotti: L'HOGGIDÌ overo il mondo non peggiore ne più calamitoso del passato. Venedig 1623. - Zu diesen Texten Bernsmeier (s. Anm. 6), S. 19-39; Marc Fumaroli: Les abeilles et les araignées. In: Anne-Marie Lecoq (Hrsg.): La Querelle des Anciens et des Modernes. Paris 2001, S. 7-220; Kristina Herrmann-Fiore: Die Freien 


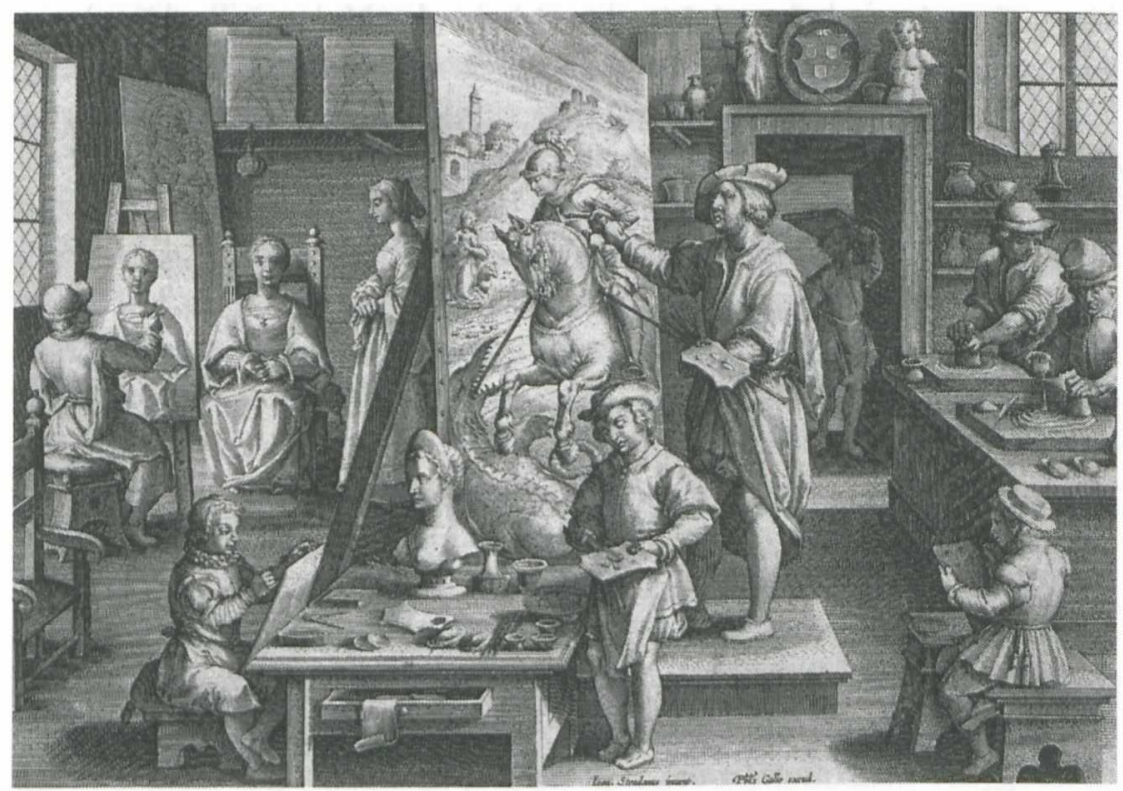

Abb. 1: Jan van der Straet: Nova Reperta, fig. 15 ('Die Erfindung der Ölmalerei durch Jan van Eyck'). 
'Fall Caravaggio' scheidet die Kunstwelt in zwei Lager: Wobei selbst seine Gegner dessen Anspruch auf radikale Neuheit ex negativo anerkennen, wenn sie behaupteten, dieser habe die Malerei(-Tradition) zerstört, oder aber ihre Angriffe gerade auf dem Argument aufbauen, seine Malerei sei doch gar nicht so neu und nur Abklatsch etwa von Giorgiones Kunst. ${ }^{11}$ Wobei mit dem hier verfolgten Akzentuieren absoluter novità natürlich nicht bestritten werden soll, daß weiterhin und parallel dazu die Antikenverehrer und die Verteidiger einer wie auch immer gearteten kreativen imitatio à la Petrarca die größte Anhängerschaft hinter sich vereinten - auch Guidiccioni vermittelt in seinem Dialog den Eindruck, Bernini würde letztlich auf diese Position einschwenken. ${ }^{12}$ Der hier allein entscheidende Punkt angesichts dieser gleichzeitigen, konkurrierend-pluralen novità-Vorstellungen bleibt, daß offenbar um 1600 konsequenter Traditionsbruch und 'voraussetzungslose' Innovation als alternative Form des Neuen denk- und vertretbar wurden.

Bezeichnenderweise wird diese Vorstellung vom Neuen bislang ausschließlich mit der Avantgarde-Bewegung der Moderne in Verbindung gebracht (natürlich unter ganz anderen historischen Vorzeichen): So wirkmächtig war aber offenbar deren Selbstpropaganda vom radikalen Bruch mit allem Vorangehenden, daß die Frage nach einer über das 19. Jahrhundert zurückreichenden Vorgeschichte dieser Idee gar nicht erst gestellt wurde. ${ }^{13}$ Und noch an einen zweiten Gesichtspunkt erinnert der Vergleich mit

Künste im Altertum und in der Neuzeit. Ein Wettstreit, dargestellt von Alessandro Tassoni im Jahre 1620. In: Victoria von Flemming und Sebastian Schütze (Hrsg.): Ars naturam adiuvans. Festschrift für Matthias Winner zum 11. März 1996. Mainz 1996, S. 381-400.

1 Verwiesen sei nur auf die klassische Studie von Louis Marin: Detruire la peinture. Paris 1977.

12 Vielleicht der interessanteste literarische Vertreter einer Theorie des überraschend Neuen aus dem Alten im frühen 17. Jahrhundert ist Giambattista Marino, vgl. etwa das auch die Bildkünste erwähnende Vorwort zu Della Lira del Cavalier Marino parte III. Venedig 1629. - Zu den Künsten (je mit weiterer Literatur) Klaus Irle: Der Ruhm der Bienen. Das Nachahmungsprinzip der italienischen Malerei von Raffael bis Rubens. Münster 1997; Robert Williams: Art, theory, and culture in sixteenthcentury Italy. From techne to metatechne. Cambridge u. a. 1998; Schlitt (s. Anm. 7); Maria H. Loh: New and Improved. Repetition as Originality in Italian Baroque Practice and Theory. In: Art Bulletin 86 (2003), S. 477-504; Cropper (s. Anm. 2).

13 Aus der umfangreichen Literatur dazu sei nur zitiert: Harold Rosenberg: The Tradition of the New. New York 1959; Rosalind Krauss: Die Originalität der Avantgarde 
der Avantgarde: So wie deren permanenter Total-Innovations-Anspruch nur als mythische Überhöhung und Stilisierung zu verstehen ist, so konnte es natürlich auch um 1600 keine 'absoluten' Innovationen geben. Die zeitgenössische Wahrnehmung von novità erweist sich so zentral als Frage der Bewertung, demonstrativen Setzung und Selbst-Mythisierung.

Vor diesem weitgreifenden Hintergrund kann mein Beitrag exemplarisch allein zwei Aspekte untersuchen: Mit Annibale Carracci steht der Künstler im Zentrum, der wie kein anderer vor ihm und bereits mit den ersten sicher für uns faßbaren Werken das eigene Euvre und Leben für die Nachwelt konsequent zu stilisieren versuchte - Carracci arbeitet von Anfang der Karriere an gezielt der Vorstellung vom 'frühbegabten Ausnahme-Künstler' und 'radikalen Erneuerer' der Kunst zu. ${ }^{14}$ Am Beispiel der Almosenspende des Hl. Rochus soll gezeigt werden, in welcher Form die Idee radikaler künstlerischer novità - innerhalb der herrschenden künstlerischen Mimesis-Doktrin und vor dem Schritt zur Abstraktion - überhaupt verwirklicht werden konnte und was dies für den Umgang mit der Bildtradition bedeutete. Die weitere Argumentation erfolgt in drei Schritten: Zunächst gilt es, den Auftrag und ursprünglichen Hängungskontext des Gemäldes und die daraus resultierenden Vorgaben an den Künstler zu präzisieren. Erst so kann in einem zweiten Kapitel deutlich werden, welche grundlegende Konzeption Annibale im Rahmen seines lebenslangen Bemühens um die Erneuerung der Malerei mit dieser historia verfolgte - nämlich eine neuartige Bildsprache christlich-katholischer Malerei im Gefolge der nach-tridentinischen Bilderdiskussion zu begründen; eine von Annibales Rochus ausgehende Bildsprache, die eine beachtliche Erfolgsgeschichte im 17. Jahrhundert verbuchen konnte. Erst vor diesem Hintergrund läßt sich - drittens und abschließend - der herausragende Stellenwert des Werkes in Annibales künstlerischem Selbstentwurf zumindest andeutungsweise verstehen.

und andere Mythen der Moderne. Amsterdam 2000 (zuerst engl. 1985); Astrit Schmidt-Burkhardt: Stammbäume der Kunst. Zur Genealogie der Avantgarde. Ber$\operatorname{lin} 2005$.

14 Zur Selbststilisierung in der Familie Carracci und bei Annibale ansatzweise Roberto Zapperi: Annibale Carracci. Bildnis eines jungen Künstlers. Berlin 1990 und Ulrich Pfisterer: 'Erste Werke' und Autopoiesis. Der Topos künstlerischer Frühbegabung im 16. Jahrhundert. In: Ders., Max Seidel (Hrsg.): Visuelle Topoi. Erfindung und tradiertes Wissen in den Künsten der italienischen Renaissance. München/Berlin 2003, S. 263-302. 
1. Die Almosenspende des $\mathrm{Hl}$. Rochus: Auftrag und ursprünglicher Kontext

Fünf Jahre, nachdem Annibale Carracci mit seiner Kreuzigung für S. Niccolo in Bologna erstmals mit einem Altarbild an die Öffentlichkeit getreten war, bemühte sich der hochambitionierte Siebenundzwanzigjährige um ein 'internationales' Publikum außerhalb der Grenzen seiner bisherigen Wirkungsorte, Bologna und Parma (wobei Parma bereits einen ersten Schritt in diese Richtung darstellte). Um so willkommener mußte Annibale daher ein Auftrag aus Reggio Emilia für ein monumentales Historiengemälde gewesen sein, das die erste entscheidende Station aus dem Leben des Hl. Rochus illustrieren sollte und für das er wohl 1587 den Auftrag erhielt (s. Abb. 2). Wie wichtig gerade diese Stadt für den junge Maler als 'Sprungbrett' wurde, läßt sich schon daraus ersehen, daß er dort noch vier weitere Werke ausführte und möglicherweise einer seiner dortigen Auftraggeber, Gabriele Bombasi, eine der wichtigen Vermittlungsinstanzen zur Familie Farnese darstellte, in deren römischem Palast Annibale dann seine Malerei von 1595/1600 an zum erstrebten Weltruhm führen sollte. ${ }^{15}$ In Bombasis römischer Familienkapelle in S. Caterina dei Funari gelang Annibale zudem mit einem Altarbild der $\mathrm{Hl}$. Margerita, formal einer Adaptation seines früheren Gemäldes für den Dom von Reggio Emilia, 1599 sein öffentlicher Durchbruch in der Papstmetropole: Angeblich rief Caravaggio angesichts dieses Werkes aus, daß er bei diesem Anblick "sterbe" und es aktuell ja doch noch "echte Maler" gebe; Caravaggios Stil veränderte sich von diesem Zeitpunkt an unter dem Einfluß Carraccis. ${ }^{16}$

Aber nicht nur Reggio Emilia und die dort geknüpften Beziehungen, sondern das Werk der Almosenspende des Hl. Rochus selbst läßt schon vor jeder genaueren Analyse seine Bedeutung für Annibale erahnen:

15 Margherita Fratarcangeli: Gabriele Bombasi. Un letterato tra Annibale Carracci e Odoardo Farnese. In: Paragone/Arte 48/15-16 (1997), S. 112-130; Daniele Benati: L'oratorio di San Rocco. Il ruolo di Reggio nella prima attività di Annibale Carracci. In: Paola Ceschi Lavagetto (Hrsg.): Il Seicento a Reggio. La storia, la città, gli artisti. Mailand 1999, S. 51-65.

16 Vgl. zusammenfassend Charles Dempsey: Caravaggio e i due stili naturalistici. Speculare contra maculare. In: Caterina Volpi (Hrsg.): Caravaggio nel IV centenario della Cappella Contarelli. Città di Castello 2002, S. 185-196. 


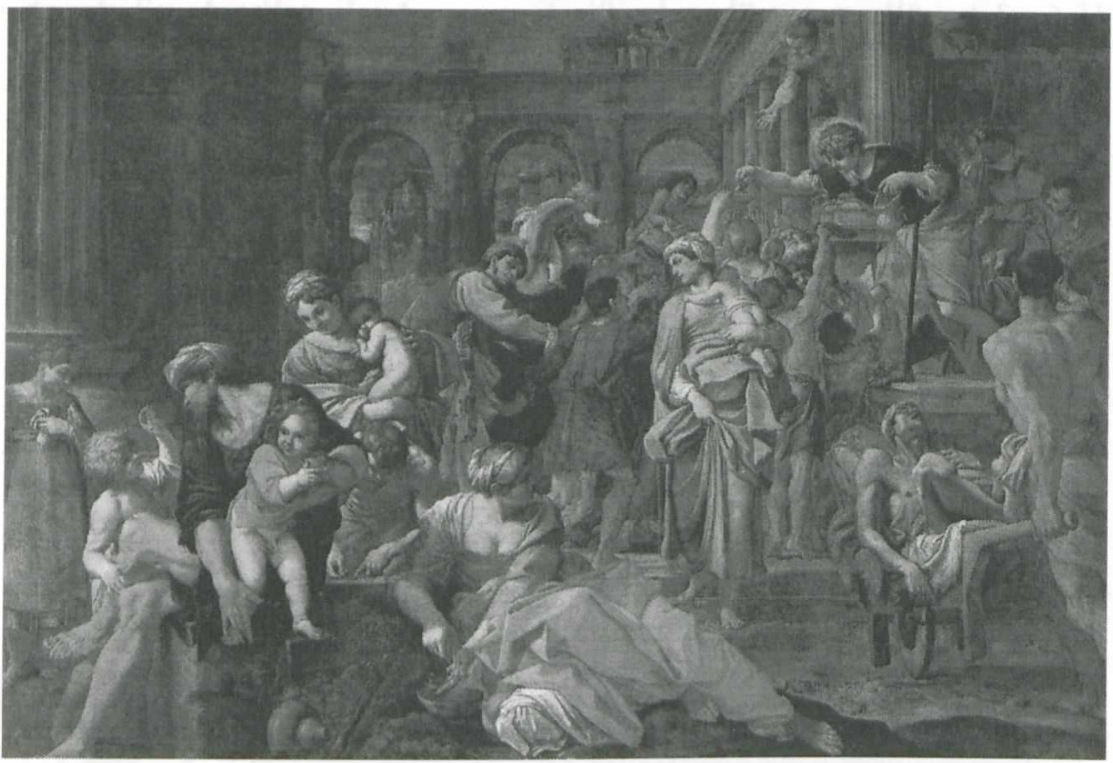

Abb. 2: Annibale Carracci: Der HI. Rochus verteilt Almosen, Dresden, Gemäldegalerie Alte Meister. 
Handelt es sich doch um das größte Leinwandgemälde, das der Maler überhaupt je ausführte, eine Fläche von 4,77 m auf 3,31 m oder rund $15,6 \mathrm{~m}^{2}$, bevölkert von über dreißig Figuren. Auch stellte Annibale, obwohl er 1594 für den Farnese-Auftrag nach Rom übergesiedelt war, noch 1595 die Almosenspende selbst fertig - andere Werke scheint er dagegen bei einer kurzen Rückkehr nach Bologna 1595 an seinen Cousin Lodovico abgetreten zu haben. ${ }^{17}$ Allein das Format und der Zeitpunkt der Vollendung lassen im $\mathrm{Hl}$. Rochus also eines der wichtigsten Bilder des Künstlers vermuten. Für die bereits von den Zeitgenossen erkannte Bedeutung des Werkes sprechen mehrere Faktoren - zunächst die zahlreichen kleinformatigen Kopien und Nachstiche, deren Liste hier um die Radierung in Melchior Küsels Icones Biblicae von 1679 erweitert sei, in denen Carraccis Komposition nun die Almosenvergabe der Apostel visualisiert (s. Abb. 3). ${ }^{18} 1660$ beschaffte sich Alfonso IV., Herzog von Modena, das Gemälde für seine Sammlung, von wo aus es 1746 beim spektakulären Verkauf der besten Werke der Este-Galerie nach Dresden gelangte. ${ }^{19}$ Zudem wurde das Gemälde nicht nur von den Kunstschrift-

17 Annibale schreibt dies selbst in einem weiter unten ausführlicher erwähnten Brief, s. Giovanna Perini (Hrsg.): Gli scritti dei Carracci. Bologna 1990, S. 155-157 (Nr. 4 f.).

18 Melchior Küsel: Icones Biblicae. Augsburg 1679, pars secunda, fig. 30; der bislang Guido Reni zugeschriebene Nachstich wird jetzt auch als Werk Francesco Brizios identifiziert, s. mit einer langen Liste weiterer Reproduktionen Veronika Birke: The Illustrated Bartsch 40 (Commentary, Part 1). New York 1987, S. 250-257 und Evelina Borea. In: Dies., Carlo Gasparri (Hrsg.): L'idea del bello. Viaggio per Roma nel Seicento con Giovan Pietro Bellori. Rom 2000, Bd. 2, S. 202 (Kat. II.2); der Stich des Baldassare Aloisi Galanini in Veronika Birke: The Illustrated Bartsch 40 (Part 2). New York 1982, S. 302 [51 (340)]; dazu und zu den Kopien in Öl auch Donald Posner: Annibale Carracci. A study in the reform of painting around 1590. London 1971, Bd. 2, S. 35-37 (Kat. 86). - Eine jüngst aufgetauchte Grisaille-Version von Annibales Rochus scheint mir mit Alessandro Brogi. In: Daniele Benati, Eugenio Riccòmini, Annibale Carracci. Mailand 2006, S. 270 f. (Kat. V.18) kein bozzetto, sondern eine Kopie zu sein.

19 Das Protokoll der Übergabeverhandlungen zwischen Bruderschaft und Herzog bei Nerio Artioli, Elio Monducci: Gli affreschi di Camillo Procaccini e Bernardino Campi in San Prospero di Reggio Emilia. Reggio Emilia 1986, S. 256; der Herzog übernahm auch das Pendant von Procaccini (s. u.); aus diesem Anlaß wurden für die Bruderschaft zwei Kopien gefertigt, die sich heute in der Pfarrkirche von Acquabona befinden. - Zum weiteren Verkauf Johannes Winkler: La vendita di Dresda. Modena 1989, v. a. S. 104 und 260 sowie Gregor J. M. Weber: Il nucleo pittorico 


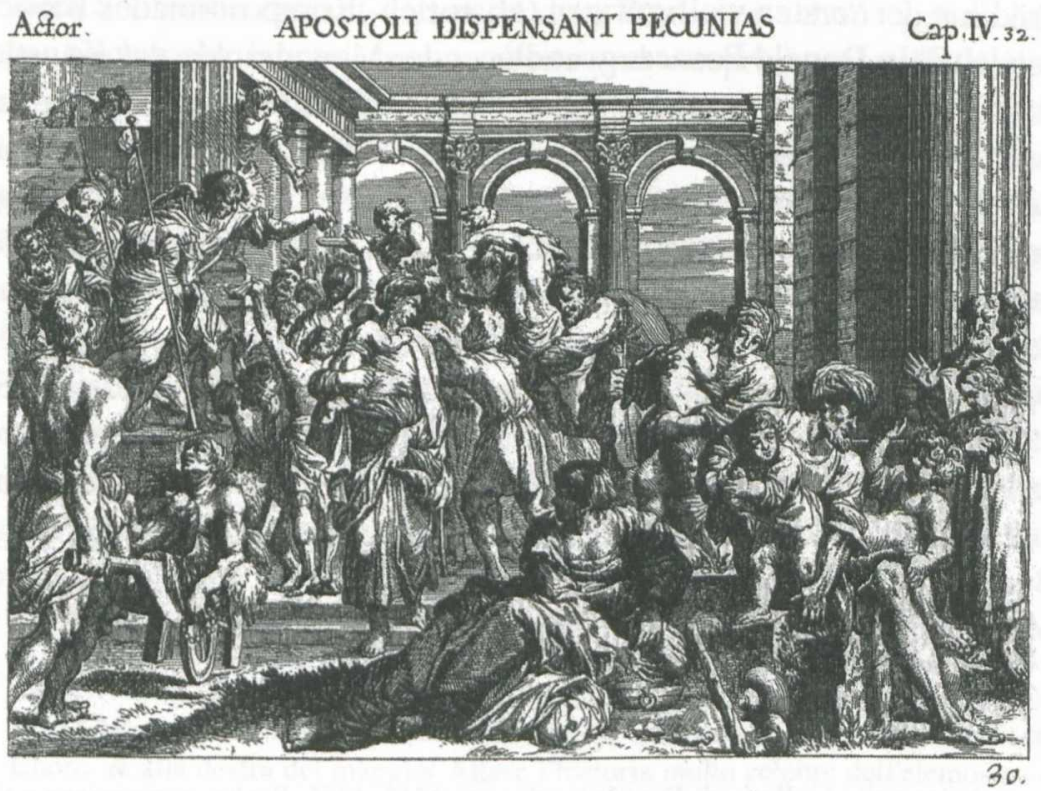

Mens eadem sancfis / amor ef propensa volnutas, Nam com mune ferief quiso $\mathrm{s}^{\prime}$ quod alfer haber.

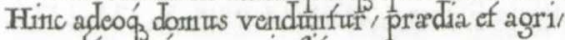
musfarumq turus sic fif acervus opum.

Inde ficef curvis pro quadra sumere parfem,

Nec minus egregio divife pauper habef.

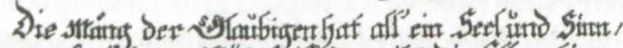

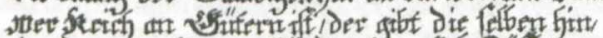

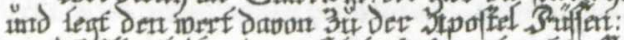

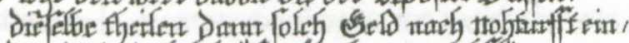

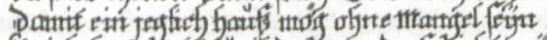

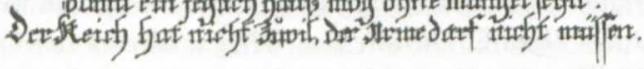

Abb. 3: Melchior Küsel: Icones biblicae. Augsburg 1679, pars secunda, fig. 30. 
stellern und Stadthistorikern des 17. Jahrhunderts hochgelobt, sondern seine Bedeutung auch 1957 von Denis Mahon aufgrund der ausgewogenklassischen Komposition und vielfältigen Affektdarstellungen mit dem Prädikat der "ersten vielfigurigen (Historien-)Komposition des Barock" geadelt. ${ }^{20}$ In Donald Posners grundlegender Monographie des Künstlers (1971) wurde nicht nur dieses Dictum wiederholt (wie im übrigen auch noch im Eintrag des Dictionary of Art von 1997), sondern offenbar auch nachhaltig festgeschrieben, daß es Annibale hier nicht um Neuerungen der Ikonographie oder Erzähltechnik, sondern ausschließlich um formal-künstlerische Probleme gegangen sei. ${ }^{21}$ Wenngleich Posners generelle Sicht des Künstlers in der Folge heftig umstritten blieb, so scheint sich doch erst jüngst mit der kurzen Erwähnung des Gemäldes in Elizabeth Croppers neuem Buch zu Domenichino und dem Problem künstlerischer novità im frühen 17. Jahrhundert eine revidierte Einschätzung anzudeuten. ${ }^{22}$ Eine eingehende Untersuchung des Gemäldes, die dessen Bedeutung im Kontext von Annibales Projekt einer Neubegründung der Malerei positionieren würde, fehlt freilich immer noch.

estense nella Galleria di Dresda negli anni 1746-1765. Scelta, esposizione e ricezione dei dipinti. In: Jadranka Bentini (Hrsg.): Sovrane Passioni. Le raccolte d'arte della Ducale Galleria Estense. Mailand 1998, S. 124-137.

20 Denis Mahon: Afterthoughts on the Carracci Exhibition. In: Gazette des BeauxArts 49 (1957), S. 193-207 und 267-298, hier: S. 282: "the first great multifigured composition of the Baroque".

21 Posner (s. Anm. 18), Bd. 1, S. 51 f., wo es unmittelbar im Anschluß an die Analyse des Rochus-Gemäldes heißt: "Annibale's works of the early 1590 s show very little concern for iconography or narrative innovations. [...]. Annibale had turned his attention more strictly to problems of pictorial form." - Vgl. an weiteren wichtigen Beiträgen außerdem Gian C. Cavalli u.a. (Hrsg.): Mostra dei Carracci. Bologna 1956, S. 207-210 (Kat. 88); Denis Mahon (Hrsg.): Mostra dei Carracci - Disegni. Bologna 1956, S. 83 f. (Kat. 105-107); Posner (s. Anm. 18), Bd. 1, S. 1 f. und Bd. 2 , S. 35-37 (Kat. 86); Anton W. A. Boschloo: Annibale Carracci in Bologna. Visible Reality in Art after the Council of Trent. Den Haag 1974, Bd. 1, S. 24 und Bd. 2, S. 187 f., Anm. 32; Patrick J. Cooney: Gianfranco Malafarino. L'opera completa di Annibale Carracci. Mailand 1976, S. 104 f. (Kat. 80); Benati (s. Anm. 15).

22 Cropper (s. Anm. 2), S. 10, 67 und 63-67, hier etwa: "It is in the Almsgiving of Saint Roch that Annibale first attributes importance to subsidiary episodes in a way that clearly anticipates a Roman grand-manner mode of narrative presentation." Dagegen lassen die kurzen Bemerkungen von Daniele Benati. In: Annibale Carracci 2006 (s. Anm. 18), S. 27 f. wenig neue Impulse erkennen. 
Die einzigen bekannten Dokumente zum Hl. Rochus stellen ein Brief Annibales (übrigens sein einziger im Original überlieferter) vom 8. Juli 1595 an die Bruderschaft und deren Antwort dar. Annibale verweist in seinem Schreiben darauf, daß er an dem Werk nun schon "sieben oder sogar noch mehr Jahren" herumarbeite und verspricht schnelle Vollendung. ${ }^{23}$ Damit würde der Zeitpunkt der Auftragsvergabe auf $1587 / 88 \mathrm{zu}$ rückdatiert, ohne daß damit zu klären ist, ob es sich beim $H I$. Rochus dann um den ersten Auftrag Annibales in Reggio handelte (seine $\mathrm{Ma}$ donna di san Matteo für die dortige Cappella dei Mercanti in S. Prospero etwa ist bereits 1588 vollendet, signiert und datiert). Von den Kunstschriftstellern des 17. Jahrhunderts hatte nur noch Francesco Scannelli 1657 die Gelegenheit, dem Bild an seinem originalen Hängungsort in der heute abgerissenen Kirche der Rochus-Bruderschaft eine enthusiastische Beschreibung zu widmen. ${ }^{24}$ Gegenstück auf der anderen Seite war eine

23 Perini (s. Anm. 17), S. 155-157 (Nr. 4 f.).

24 Francesco Scannelli: Il Microcosmo della Pittura. Hrsg. von Rosella Lepore. Bologna 1989, S. 338 f. (Reprint der Originalausg. 1657): "in Reggio stà in S. Prospero l'Altare del Choro, e nella Confraternità di S. Rocco la Tavola similmente del Choro, \& alla destra del maggior Altare l'historia molto celebre dell'elemosina di questo Santo, e se bene gli altri citati siano di bellezza, e perfettione straordinaria, sono in fatti però queste due operazioni il fiore de' più esquisiti dipinti, c'habbia mai dimostrato il medesimo Annibale. Fece attione degna di lode, e di memoria il Glorioso S. Rocco nel dispensare le proprie facoltà a' poveri, e quivi appare, come al vivo rappresentato dal raro pennello di così egregio Artefice, il quale in un tal caso altrettanto prodigo della virtù comparte a mendici della Professione continuamente in abbondanza i più rari, e qualificati effetti della Pittura, ed historia tale è una di quelle grandi, e straordinarie operazioni, le quali per contenere ogni sorte di più rari oggetti, dimostrano come un'aggregato del tutto, che la maggior eccellenza dell'arte può manifestare ad imitatione della ben disposta natura. Quivi l'inventione è rara, la disposizione molto sufficiente, l'attitudini singolari, ed i concetti, e pensieri diseminati e spiritosi, che oltre il rappresentare adequatamente ogni minima parte, danno motivo di gustosa maraviglia al riguardante, posciache oltre il Santo tutto spirito frà molti, e differenti pitocchi ciascheduno in un tal caso si palesa del tutto intento coll'arte propria per ottenere la desiata elemosina; alcuni procurano con la forza avanzarsi, altri col dimostrarsi in varie guise più bisognosi, e compassionevoli, \& in ordine a ciò non mancano gesti più efficaci, e maggiormente spiritosi, né deformità horrende, e vestiti capricciosi, e stravaganti, e quelli, che per se soli non sono bastevoli uniti con altri s'ingegnano a tutto potere di rappresentarli in sito, e forma meritevole. In somma il tutto è così bello, \& ogni particolare di tanta eccellenza, che ricoperto con maniera della più facile, e vera operatione fa conoscere un concerto d'historia senza difficoltà delle più naturali, e belle, che possa in 
gleichgroße Darstellung des Camillo Procaccini, die den Hl. Rochus beim Niederschlagen einer Pestepidemie zeigt - ein Gemälde, das 1746 ebenfalls nach Dresden verkauft wurde, allerdings im Zweiten Weltkrieg verbrannte (s. Abb. 4). ${ }^{25}$ Alle späteren Autoren - Bellori, Malvasia u. a. stifteten dann durch falsche Angaben nur Verwirrung, so soll es sich etwa beim Auftraggeber eigentlich um einen Kanoniker Brami gehandelt haben, und das Gemälde sei für S. Prospero bestimmt gewesen. ${ }^{26}$ Vor allem wird auch behauptet, Procaccini habe sein Gemälde nachträglich in Konkurrenz zu Carracci angefertigt. Dabei belegt der erhaltene Auftrag zu Procaccinis Werk eindeutig, daß es im Gegenteil zuvor zwischen 1585 und 1587 entstand, Procaccini übersiedelte dann nach Mailand. ${ }^{27}$ Carracci übernimmt sozusagen als 'zweite Wahl' (oder aber - was die Bewertung grundlegend ändern würde - als nachträglicher, dafür umso erfolgreicherer Konkurrent Procaccinis) dessen Auftrag für das zweite Gemälde, die Almosenspende des Hl. Rochus.

Das genaue Aussehen der im 19. Jahrhundert abgerissenen Bruderschafts-Kirche läßt sich heute nurmehr annähernd auf der Grundlage eines Grundrisses des 19. Jahrhunderts ermitteln (s. Abb. 5) ${ }^{28}$ Für die

alcun tempo la forza de' pennelli rappresentare a buoni intelligenti; e di questa particolar' historia si compiacque sì fattamente il famoso Guido Reni, che dopo haverla co' fatti, e parole più volte encomiata incitato dal proprio gusto non mancò d'eternarla a tutto potere col mezo della stampa d'acqua forte, dimostrando con una tal' insolita attione essersi compiaciuto in estremo di questo raro dipinto; [...]." - Vgl. außerdem noch die kurze Erwähnung bei Fulvio Azzari: Compendio dell'Historie della città di Reggio [...]. Reggio Emilia 1623.

Zu Procaccinis Gemälde und den erhaltenen Vorstudien Nancy W. Neilson: Camillo Procaccini. New York 1979, S. 10-17, 26 f. und 61-63; Massimo Pirondini, Elio Monducci: La Pittura del Cinquecento a Reggio Emilia. Mailand 1985, S. 178-182; Benati (s. Anm. 15).

26 Giovan Pietro Bellori: Le vite de' pittori, scultori e architetti moderni. Hrsg. von Evelina Borea. Turin 1976, S. 28 f.; Carlo Cesare Malvasia: Felsina Pittrice. Le vite de' pittori bolognesi. Hrsg. von Giampietro Zanotti. Bologna 1841, Bd. 1, S. 291 f.; diese Irrtümer dann offenbar definitiv festgeschrieben durch Giuseppe Campori: Gli artisti italiani e stranieri negli stati Estensi. Modena 1855, S. 386.

27 Abgedruckt in Nerio Artioli, Elio Monducci: Gli affreschi di Camillo Procaccini e Bernardino Campi in San Prospero di Reggio Emilia. Reggio Emilia 1986, S. 255.

28 Der Abriß der Kirche erfolgte erst Mitte des 20. Jahrhunderts; auf einer Zeichnung des 19. Jahrhunderts fußend der Grundriß bei Elio Monducci, Vittorio Nironi: Arte e storia nelle chiese reggiane scomparse. Reggio Emilia 1976, S. 89-92. Dort ist 


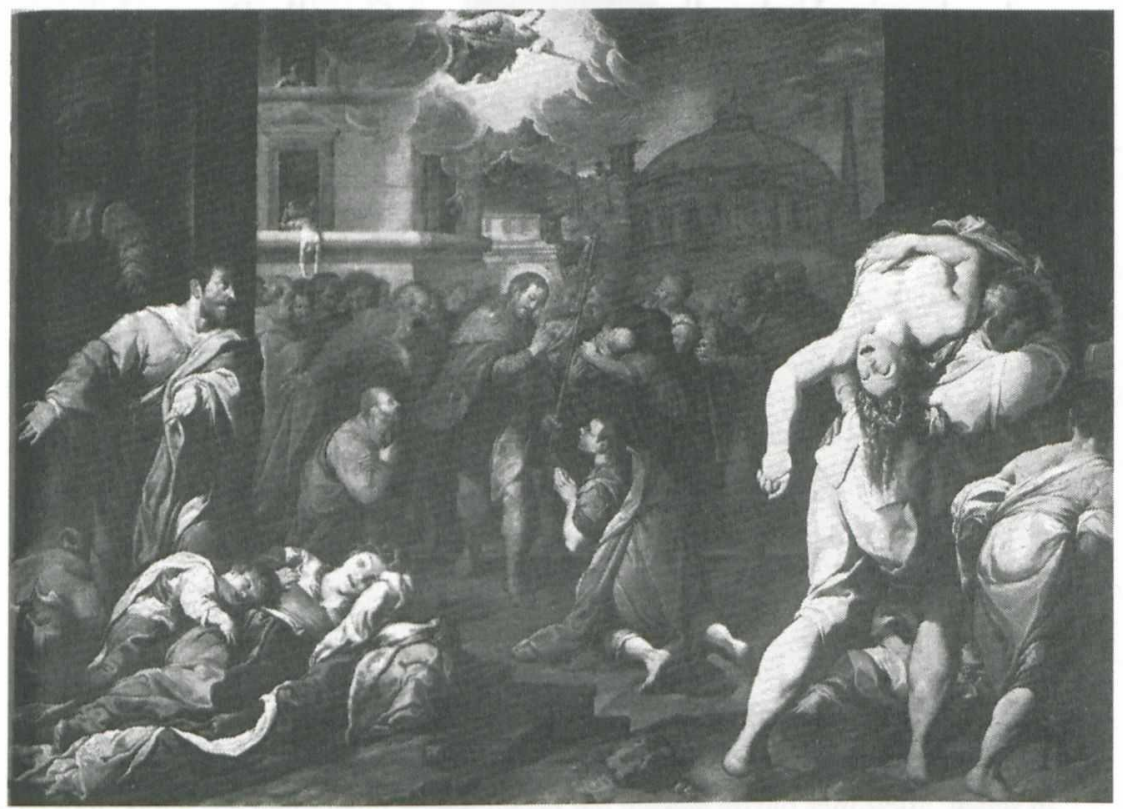

Abb. 4: Camillo Procaccini: Der Hl. Rochus schlägt eine Pestepedemie nieder, ehem. Dresden, Gemäldegalerie. 
beiden monumentalen Gemälde kommt allerdings nur ein Aufhängungsort in Frage: nämlich die Wandflächen zuseiten des Hauptaltars, wie es auch schon im Verhandlungsprotokoll mit Camillo Procaccini vom 19. August 1585 festgeschrieben wurde: "per servizio al destro e sinistro latto del altare grande". ${ }^{29}$ Was bringen diese Überlegungen nun an weiterführenden Einsichten? Zunächst eine indirekte: Annibales Gemälde entstand nach dem Werk Procaccinis für dieselben Auftraggeber, daher dürften die bereits für Procaccini gültigen Kriterien zumindest ähnlich auch bei Carracci angelegt worden sein.

Für Procaccinis Komposition haben sich nicht nur zwei Entwurfszeichnungen, sondern auch ein Bozzetto in Öl erhalten (s. Abb. 6). ${ }^{30}$ Diese sehr genau ausgearbeitete Vorstudie diente den Auftraggebern zur Kontrolle und Genehmigung des Entwurfs, wie es sich für Bozzetti der Jahre um und nach 1600 vielfach nachweisen läßt. ${ }^{31}$ Trifft dies zu, dann sind die Abänderungen der ausgeführten Fassung besonders aufschlußreich: Ergänzt wurde erstens in einem aufreißenden Wolkenloch

die Kirche zwar nach der vollständigen Umgestaltung 1761 durch Andrea Tarabusi zu sehen; allerdings dürfte sich an der prinzipiellen Plazierung der Altäre und damit auch an den freien Wandflächen nichts Grundlegendes geändert haben. Über den beiden Seitenaltären befanden sich Gemälde von Palma il Giovane, eine Madonna mit den Hl. Rochus und Martin sowie einem Stifter bzw. eine Kreuzigung. Die Bruderschaftskirche unterstand der Benediktinerabtei S. Prospero.

29 Artioli, Monducci 1986 (s. Anm. 27), S. 255. - Zu Tradition und spezifischen Anforderungen solcher quadri laterali s. Michael Matile: Quadri laterali im sakralen Kontext. Studien und Materialien zur Historienmalerei in venezianischen Kirchen und Kapellen des Cinquecento. München 1997.

30 Siehe Anm. 25.

31 Zum Procedere (in dessen dokumentiertem Verlauf allerdings nur Zeichnungen, kein bozzetto erwähnt wird) und den Sachverständigen vgl. das bereits zitierte Verhandlungsprotokoll bei Artioli, Monducci 1986 (s. Anm. 27), S. 255: "Che havendo visto i disegni datti da messer Camillo Procaccini, qual contengono una parte della vitta di S. Rocho et considerato che sarebbino buoni da metere in tella per servire a questa nostra chiesa di $\mathrm{S}$. Roccho per honorarla al meglio che si può, et havendo havuto d'essi il parere del signor Lelio Orsi da Novalara, dal Pilla et hora dal molto magnifico signore Gabrielo Bombacio, dal ilustre signor conte Lelio Rugieri et d'altri, si pensò che sarebbe bene convenisse con esso Proccasino per eseguirgli in opera." - Zusammenfassend zur Funktion von bozzetti zuletzt Oreste Ferrari: Causes et conditions de l'exécution de bozzetti dans la peinture romaine du XVIIe siècle. In: Les cieux en gloire. Paradis en trompe-l'oeil pour la Rome baroque. Ajaccio 2002, S. 53-64. 


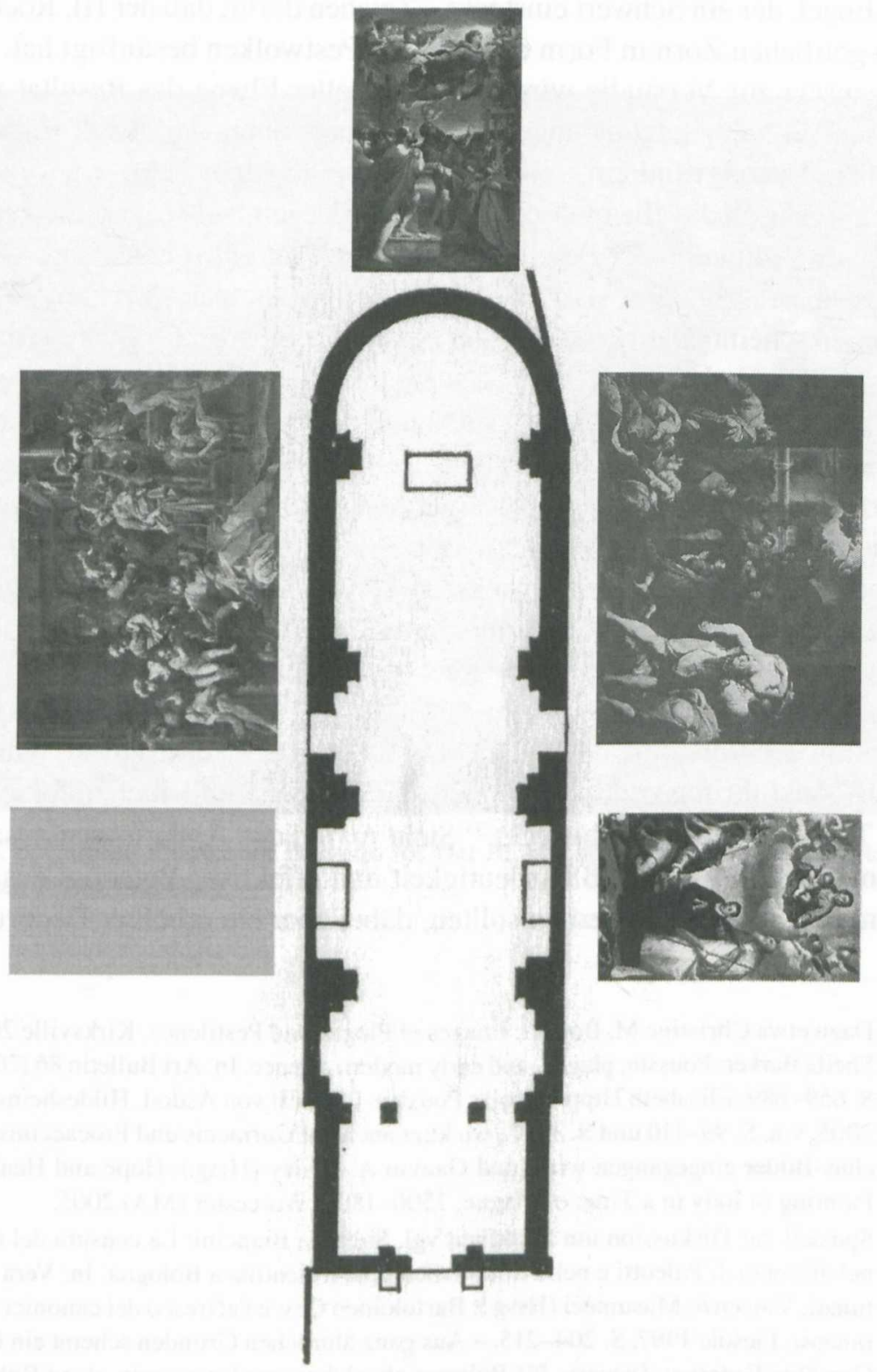

Abb. 5: Rekonstruktionsskizze (nicht maßstäblich) der Bildausstattung der Kirche der Rochus-Bruderschaft, Reggio Emilia: Neben den Gemälden von Carracci und Procaccini waren über den Seitenältaren zwei Gemälde von Palma il Giovane zu sehen (eines davon eine heute verschollene Kreuzigung). 
ein Engel, der ein Schwert einsteckt - Zeichen dafür, daß der Hl. Rochus den göttlichen Zorn in Form der dunklen Pestwolken besänftigt hat. Im Gegensatz zur Vorstudie wird so auf visueller Ebene das Resultat von dessen Wirken eindeutig angezeigt. Dazu paßt auch, daß in der Endversion im Hintergrund ein Leichnam einfach aus dem Fenster geworfen wird, wodurch die alle menschlichen Grundregeln außer Kraft setzende Ausnahmesituation der Pest noch expliziter vorgeführt erscheint. Allerdings überrascht dann, daß im Vordergrund genau das Gegenteil zu geschehen scheint: Das bereits aus der Antike als Beispiel für die Grausamkeit der Pest bekannte Beispiel des Kleinkindes, das noch an der Brust der toten Mutter nach Nahrung sucht und daher selbst sterben wird, dieser spätestens seit Raffaels Il Morbetto in der Frühen Neuzeit allgegenwärtige Bildtopos wird in Procaccinis Ausführung dadurch stark zurückgenommen, daß die Mutter nun vollständig angezogen daliegt und das Kind nicht mehr nach der Brust greift. ${ }^{32}$ Verständlich wird diese eigentlich 'verunklärende' Abänderung, betrachtet man ergänzend die - vermutliche - Familiengruppe zur Rechten, wo ein Mann seine tote Frau kopfüber auf den Rücken geladen zu Grabe trägt. Ist dieser Toten das Gewand in der Vorstudie noch ganz vom Oberkörper gerutscht, so hält sie in der Ausführung zumindest eine Brust mit Hand und Tuchzipfel selbst im Tode einigermaßen bedeckt. ${ }^{33}$ Sieht man diese Änderungen zusammen, scheint es fast, daß Eindeutigkeit und affektives Potential des Ereignisses intensiviert werden sollten, dabei aber ein erhöhter Decorum-

32 Dazu etwa Christine M. Boeckl: Images of Plague and Pestilence. Kirksville 2000; Sheila Barker: Poussin, plague, and early modern science. In: Art Bulletin 86 (2004), S. 659-689; Elisabeth Hipp: Nicolas Poussin. Die Pest von Asdod. Hildesheim u. a. 2005, v. a. S. 99-110 und S. 319 f., wo kurz auch auf Carraccis und Procaccinis Rochus-Bilder eingegangen wird; und Gauvin A. Bailey (Hrsg.): Hope and Healing. Painting in Italy in a Time of Plague, 1500-1800. Worcester (MA) 2005.

33 Speziell zur Diskussion um Nacktheit vgl. Stefania Biancini: La censura del nudo nel discorso di Paleotti e nella trattatistica post-tridentina a Bologna. In: Vera Fortunati, Vincenzo Musumeci (Hrsg.): Bartolomeo Cesi e l'affresco dei canonici lateranensi. Fiesole 1997, S. 204-215. - Aus ganz ähnlichen Gründen scheint ein PestGemälde Federico Zuccaris für Bologna abgelehnt worden zu sein, dazu Roberto Zapperi: Federico Zuccari censurato a Bologna dalla corporazione dei pittori. In: Städel-Jahrbuch, N. F. 13 (1991), S. 177-190 und Tristan Weddigen: Federico Zuccari zwischen Michelangelo und Raffael. Kunstideal und Bilderkult zur Zeit Gregors XIII. In: Ders. (Hrsg.): Federico Zuccaro. Kunst zwischen Ideal und Reform. Basel 2000, S. 195-269. 


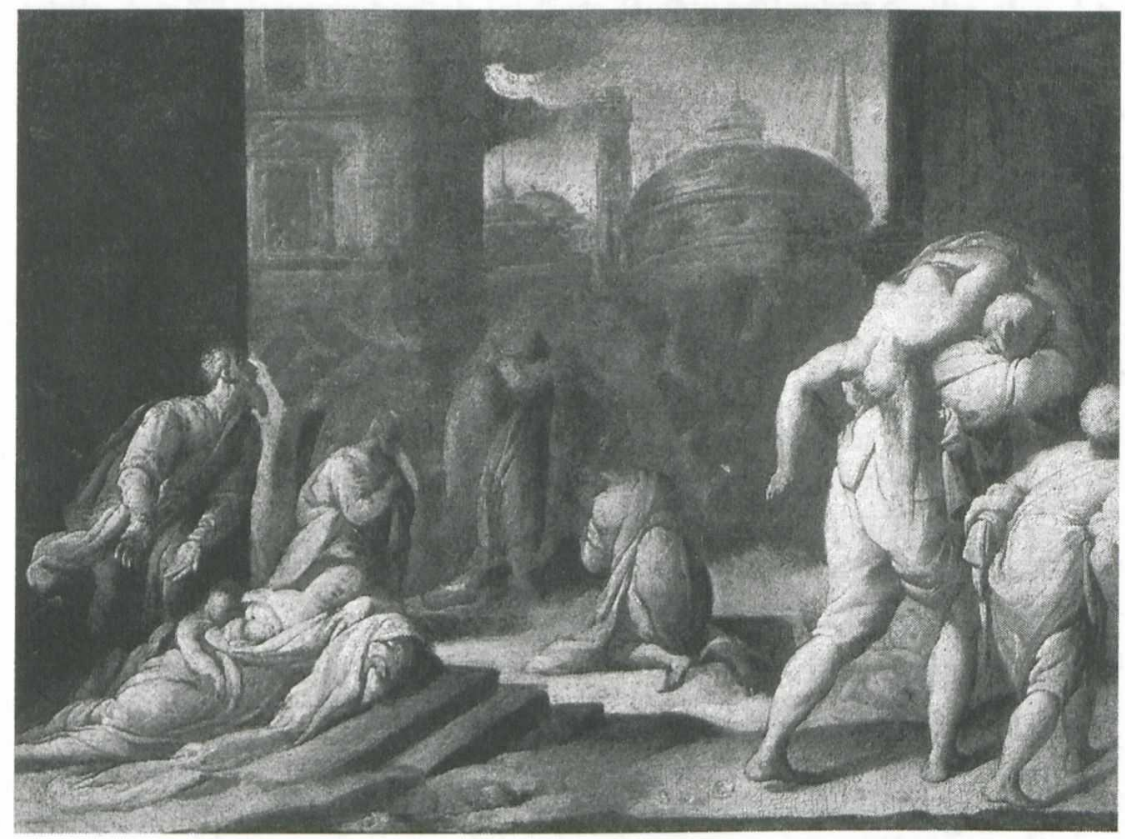

Abb. 6: Camillo Procaccini: Bozzetto für Der Hl. Rochus schlägt eine Pestepedemie nieder, Privatsammlung. 
Anspruch verbot, allzu viel nackten, insbesondere weiblichen Körper zu zeigen. Und noch ein dritter Aspekt läßt sich erkennen: Die Gestik der stehenden Männerfigur links, bei der es im bozzetto nicht ganz klar scheint, ob sie den Heiligen auf die verstorbene Familie zu ihren Füßen oder aber auf die Gläubigen vor dem Bild hinweisen will, wird in der Ausführung so präzisiert, daß sich nun eindeutig die Gläubigen und Bruderschaftsmitglieder vor dem Bild angesprochen fühlen durften - Bild und Betrachter treten so in noch stärkere, Realitäts-Grenzen überspielende Beziehung. Eindeutigkeit der Bildaussage, Decorum und affektive Wirkung stellen nun aber bekanntlich drei zentrale Forderungen des gegenreformatorischen Bildverständnisses dar. ${ }^{34}$ Annibales Auftraggeber waren offenbar um eine sehr durchdachte Umsetzung der Vorgaben des Trienter Konzils und der zeitgenössischen Diskussionen zu einem legitimen katholischen Bildgebrauch bemüht. Anbringungskontext und Art des Auftrags sowie die prestige-trächtige Größe des Gemäldes mußten Annibale daher gerade dieses Werk als ideale Möglichkeit erscheinen lassen, seine Vorstellungen von der neuen christlich-katholischen Malerei zu verwirklichen.

Erst vor dem Hintergrund der Analyse von Procaccinis bozzetto und Gemälde gibt sich schließlich noch ein weiteres Problem zu erkennen: Laut Scannelli hing Annibales Leinwand 1657 “zur Rechten des Altares" - will sagen: an der linken Wand für den zum Altar Hinblickenden. ${ }^{35}$ Wie sich noch zeigen wird, entspricht dieser Plazierung die (an Correggio und Veronese orientierte) Schrägkomposition von Carraccis Almosenvergabe und die Ausrichtung nach links auf die Gläubigen vor dem Bild hin. Um genau diese Ansprache und Ausrichtung war es jedoch zumindest ansatzweise auch Procaccini zu tun, wie die Gestik des Mannes im linken Bildvordergrund zu belegen scheint - weshalb Procaccinis Gemälde eigentlich ebenfalls für die linke Wand gedacht gewesen sein müßte. Dieser Ort dürfte tatsächlich die prominentere Hängungsmöglichkeit

Zusammenfassend Christian Hecht: Katholische Bildertheologie im Zeitalter von Gegenreformation und Barock. Studien zu den Traktaten von Johannes Molanus, Gabriele Paleotti und anderen Autoren. Berlin 1997.

$35 \mathrm{DaB}$ Scannellis Formulierung so zu verstehen ist, zeigt etwa seine entsprechende Beschreibung der Putten-Fresken des Guido Reni in S. Maria dei Servi, Bologna, die sich in einer Kapelle des linken Seitenschiffs befanden: "in una Capella alla destra dell'Altare maggiore” (Scannelli [Anm. 24], S. 350). 
gewesen sein, da sie von annähernd südlichem Sonnenlicht beleuchtet wurde und zudem jeder von der Straße durch das Seitenportal Eintretende den Blick unweigerlich in diese Richtung lenkte. Sollte also Annibale nicht nur von Procaccini den zweiten Gemäldeauftrag übernommen, sondern auch dessen Werk nachträglich vom besseren Hängungsort verdrängt haben?

\section{Annibales Bildsprache zwischen historia und schemata virtutum}

Betrachten wir Annibales Gemälde genauer (s. Abb. 2): Die Szenerie öffnet sich auf den monumentalen Vorhof des Palastes, den der jugendliche Rochus von seinen Eltern geerbt hat. Der neue Besitzer und zukünftige Heilige steht auf einem Postament erhöht und verteilt das geerbte Geld an eine große und drängende Menschenmenge, deren Hände sich aus allen Richtungen den Geldstücken entgegenrecken. ${ }^{36}$ Aus dieser Menschenmenge stechen vier Gruppen heraus: ein muskulöser Mann fährt in einer Schubkarre einen kranken Bettlägrigen heran. Auf der anderen Seite wird ein blinder Geigenspieler von seinem jungen Begleiter zum Geschehen geführt. In der Mitte schließlich kommt dem Betrachter eine junge Mutter mit ihrem Kleinkind auf dem Arm entgegen, die bereits ihr Almosen erhalten hat und sich wohl auf dem Weg zu der Menschengruppe im linken Vordergrund befindet: Dort haben sich einige der bereits beschenkten Bedürftigen versammelt: Vermutlich eine Waise hält ihr Beutelchen mit der Aussteuer vor der Brust, daneben freut sich eine Familie über die unverhofften Gaben, schließlich zählen zwei weitere Personen ihre Geldstücke ab; möglicherweise ist zumindest die sitzende Frauengestalt durch den Wanderstab und die Trinkflasche als Pilgerin zu verstehen. ${ }^{37}$

36 Zur Ikonographie etwa Venezia e la Peste 1348/1797. Venedig 1979; San Rocco nell'arte. Un pellegrino sulla via Francigena. Mailand 2000 und Werner Roemer: Sankt Rochus. Die Verehrung des Heiligen in Kunst und Geschiche. Kevelaer 2000.

37 Alle Nachstiche zeigen hinter der stehenden Waise noch zwei im Gemälde selbst nicht erscheinende Männer, die über das Tun des Rochus und seinen Sinn zu diskutieren scheinen; da an dieser Stelle die Oberfläche des Dresdener Gemäldes gestört scheint, wurden diese möglicherweise übermalt oder entfernt. - Zur herausragenden Bedeutung der Unterstützung junger Mädchen vgl. etwa Iulius Roscius 
Dieser kompositionelle Aufbau, der vor der Hintergrundsfolie einer wenig differenzierten Menschenmenge verschiedene Figurengruppen von Bedürftigen isoliert herausstellt, wurde immer schon wahrgenommen - teils als entscheidendes kompositorisches Element auf dem Weg zu Annibales 'römischem (klassischem) Stil' gelobt, teils aber auch mit der langen Arbeit an dem Werk zu erklären versucht und damit implizit kritisiert. Nun beherrscht Annibale die Kunst der Komposition um 1595 freilich so souverän, daß man die so offensichtliche Isolierung der Gruppen kaum als unglückliches Resultat aus einer sukzessiven und eilig abgeschlossenen Fertigung verstehen will, bei der die Einzelstudien dem Gesamt nicht wirklich überzeugend integriert wurden. Ebenso wenig läßt sich jedoch diese Art der Komposition allein mit einer formal-stilistischen 'Entwicklung' erklären: In Domenichinos Fresko der Almosenspende der Hl. Cäcilia etwa (von 1613/14; s. Abb. 7), dessen Gesamtanlage unzweideutig vom Modell Carraccis ausgeht, scheint nicht nur die Integration der Nebengruppen ganz anders gelöst. Bereits Malvasia kritisierte, daß Domenichino im Gegensatz zu Carracci unpassend ablenkende Szenen eingefügt habe. ${ }^{38}$

Worin besteht also das Besondere von Annibales Lösung? Einen ersten Anhaltspunkt kann die Diskussion des späteren 16. Jahrhunderts über Darstellungsmöglichkeiten und über das Verhältnis von konkreten Szenen der Heilsgeschichte bzw. von Heiligenleben einerseits, von abstrakten christlich-theologischen Konzepten andererseits liefern. So zeigt sich - um mit einem Beispiel der Praxis zu beginnen - Federico Barocci 1574/75 zunächst zögerlich, als er den Auftrag der Misericordia-Bruderschaft von Arezzo erhält, ein Gemälde des (abstrakten) "mistero della misericordia" zu fertigen. Dem Maler scheint dies ein "unangebrachtes Thema, um ein schönes Bild zu machen”, er schlägt dagegen eine "Verkündigung, Himmelfahrt, Heimsuchung oder andere historia" vor. Auch wenn Barocci letztlich einlenkte, wie die ausgeführte Version einer Madonnenerscheinung über Arezzo zeigt, so werden doch ereignishafte

Hortinus: Icones operum misericordiae. Rom 1586, S. 18 (zu “Operire nudos”): “In quo genere nunquam satis laudari potest eorum beneficentia, qui puellas ob egestatem vix se tegi valentes, huiusmodi donant pietatis munusculis: ex quo fit, ut \& sanitati corporis, \& multo magis animarum saluti periclitanti subveniant."

38 Richard E. Spear: Domenichino. New Haven/London 1982, S. 180 f. (Kat. 42.ii.); Cropper (s. Anm. 2), S. 67 f. 


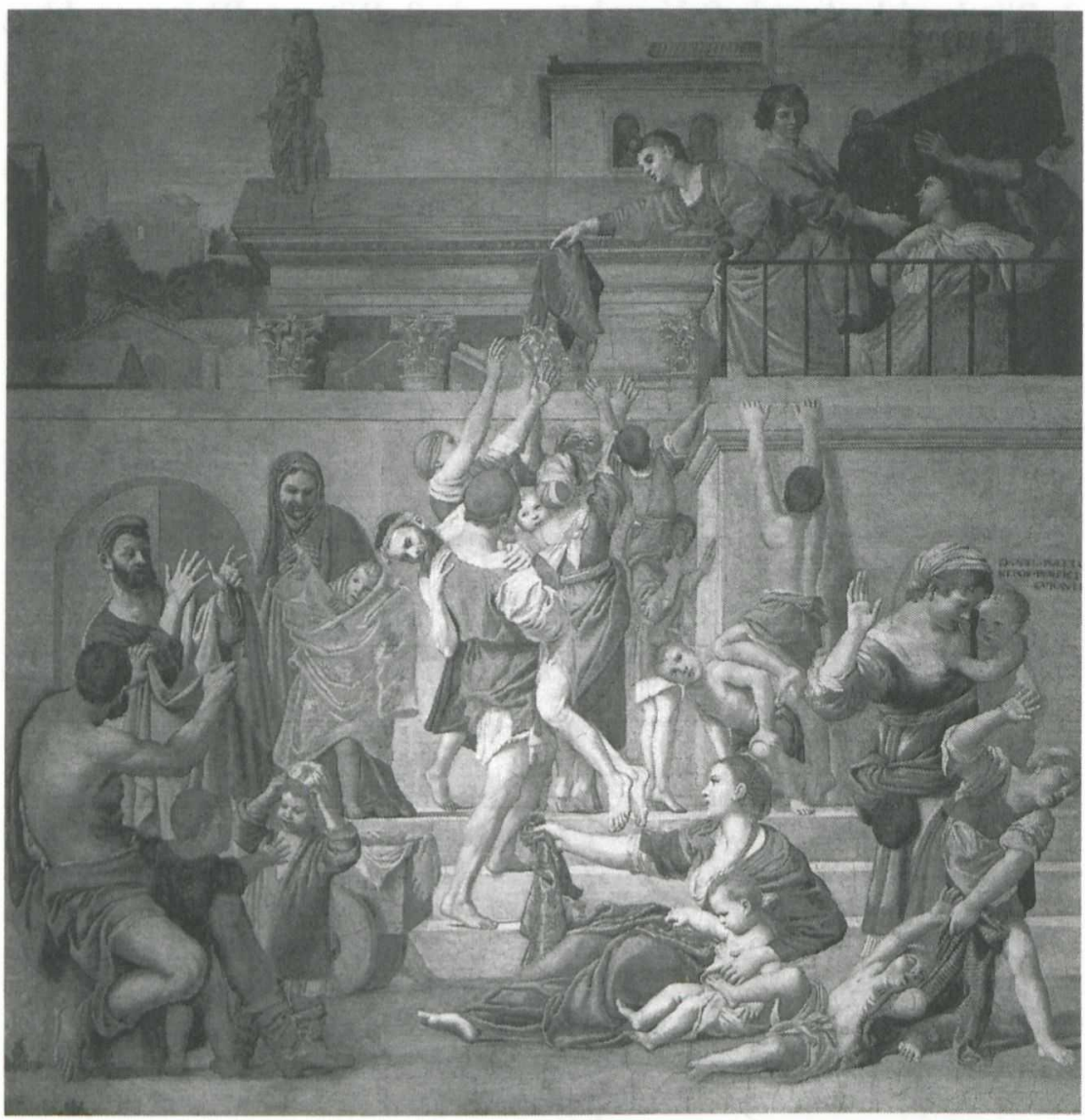

Abb. 7: Domenichino: Almosenspende der Hl. Caecilia, Rom, St. Luigi dei Francesi. 
Elemente integriert: Ähnlich wie bei Carracci erhalten am Rande des Geschehens die drei Grundtypen der Bedürftigen: 1. temporär Kranke, 2. Blinde, d.h. dauerhaft Versehrte, sowie 3. Witwen, Waisen und bedürftige Familien, kurz: poveri vergognosi Unterstützung. ${ }^{39}$ Entsprechend - und nun auf der Ebene der Theorie - kommentiert Gabriele Paleotti in seinem Discorso intorno alle imagini sacre e profane (1582) die Schwierigkeit, theologisch korrekte Gemälde von Tugenden und Lastern zu erstellen: Am besten exemplifiziere man die gewünschten abstrakten Eigenschaften am Beispiel konkreter Personen, bevorzugt der Heiligen - wogegen Paleotti bemerkenswerterweise die wenig später von $\mathrm{Ce}$ sare Ripa so erfolgreich praktizierte Lösung 'Personifikation' als “cosa comune e triviale" ablehnt. ${ }^{40}$

Für Annibales Rochus läßt sich eine analoge 'Überblendungstechnik' vermuten (allerdings mit dem Unterschied, daß Paleotti mehr an die traditionellen exempla virtutis herausragender Personen denkt, wogegen Carracci die 'unbedeutenden Nebenpersonen' zu Tugendchiffren formt). Jedenfalls sollte dann nicht nur eine Station aus dem Leben des

39 Andrea Emiliani: Federico Barocci (Urbino 1535-1612). Bologna 1985, Bd. 1, S. 128-149, hier: S. 129 die entscheidenden Passagen des Briefwechsels; am 5. Nov. 1574 antwortet der Maler aus Urbino: "[...] il voler fare il mistero della Misericordia non pare a me che sia sugietto troppo a proposito per fare una bella tavola, e non ci curando le S. V. che si facessi altro misterio purché fusse della gloriosa vergine ve sariano altre istorie più a proposito con piú belle inventione come sarebbe la Anuntiata la Sumptione la Visitazione o altre istorie [...]."

40 Gabriele Paleotti: Discorso intorno alle imagini sacre e profane. In: Paola Barocchi (Hrsg.): Trattati d'arte del Cinquecento fra manierismo e controriforma, Bd. 2. Bari 1961, S. 117-509, das relevante Kapitel S. 452-466, hier etwa S. 460: "Ma di più: per fare queste pitture delle virtù, potrà talora l'accorto pittore valersi delle imagini di persone onorate, massime de' santi, cavendone sensi buoni e accommodandole al' proposito suo: [...]." Vgl. auch ebd., S. 294-296. - Zuletzt dazu Holger Steinemann: Eine Bildtheorie zwischen Repräsentation und Wirkung. Kardinal Gabriele Paleottis "Discorso intorno alle imagini sacre e profane" (1582). Hildesheim u. a. 2006. - In umgekehrter Argumentation glaubte dagegen Molanus 1570 in einem eigenen Kapitel seines Bildertraktates widerlegen zu müssen, daß die Kirche bestimmte Tugenden, insbesondere Fides, Caritas und Spes, zu vermeintlichen Heiligen 'vermenschlicht' habe (ohne dabei die Erzählung der Legenda Aurea von Sapientia und ihren drei Töchtern dieses Namens zu bestreiten). Vgl. Johannes Molanus: Traité des saintes images. Hrsg. von François Bœspflug u. a. Paris 1996, Bd. 1, S. 305-307, Bd. 2, S. 210-214 (Ecclesiam non convertisse schemata virtutum in fictitos Sanctos. Cap. LXI). 


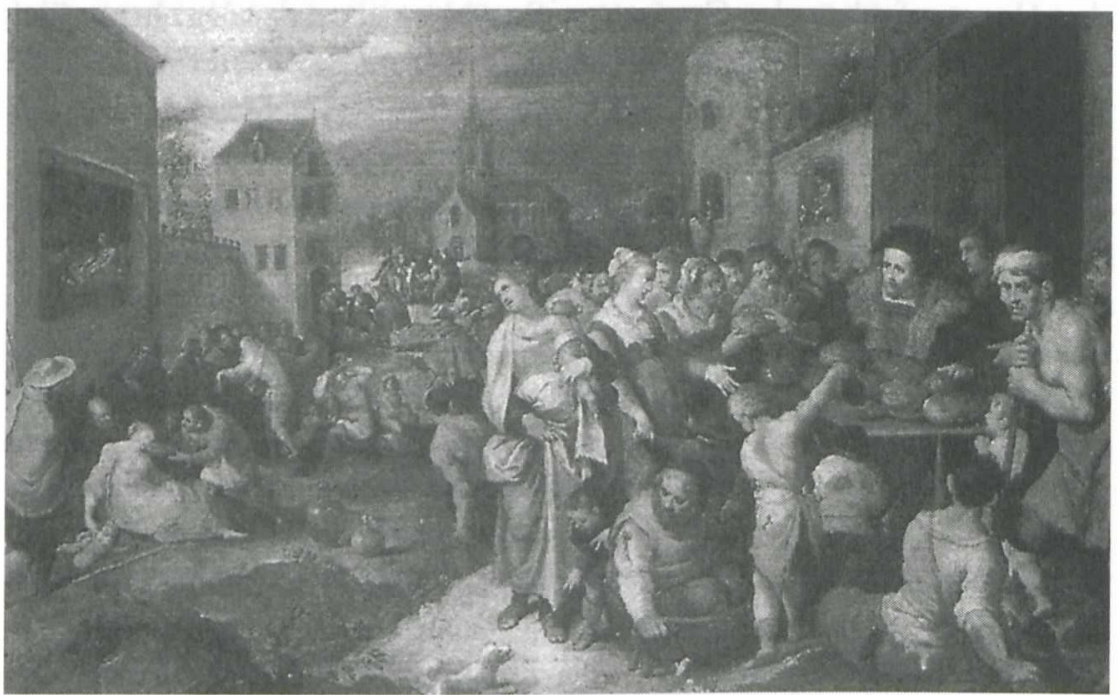

Abb. 8: Frans Francken II.: Sieben Werke der Barmherzigkeit, Privatsammlung. 
Titelheiligen dargestellt werden, sondern zugleich die für die Bruderschaft zentralen Tugenden, voran Mitleid und Nächstenliebe: Denn zu den Hauptaufgaben der Confraternità zählte neben der Verehrung des Heiligen selbstverständlich das weite Spektrum der Werke der Barmherzigkeit mit einem Schwerpunkt auf der Sorge für Seuchenkranke. ${ }^{41}$ Daß Annibales Gemälde tatsächlich von den Zeitgenossen als Darstellung karitativer Tugenden unter dem Mantel einer exemplarischen Heiligenszene verstanden wurde, verdeutlicht die Übernahme der zentralen Mutterfigur Annibales 1615 in ein Gemälde Frans Franckens II. eben zu den Sieben Werken der Barmherzigkeit (s. Abb. 8): Da Annibales Komposition, wie gesehen, unmittelbar nach Fertigstellung durch mehrere Stiche und kleinformatige Kopien in ganz Europa bekannt gemacht wurde, braucht das Zitat in einem niederländischen Werk nicht zu verwundern. ${ }^{42}$ Für die Betrachter von Annibales Gemälde wäre es jedenfalls selbstverständlich gewesen, die zentrale Mutter mit Kind als Chiffre für Caritas zu verstehen, war dieses Sinnbild doch seit Jahrhunderten im visuellen Gedächtnis verankert. ${ }^{43}$ Die Bedeutung der Figur wird dem

${ }^{41}$ Vgl. allgemein Pio Paschini: La Beneficenza in Italia e le 'Compagnie del Divino Amore'. Rom 1925; Giovanni Mantese: Il Card. Agostino Valier e l'origine delle Compagnie della Carità. In: Archivio Veneto, 5. ser., 90 (1972), S. 5-26; Brian Pullan: "Support and redeem". Charity and poor relief in Italian cities from the fourteenth to the seventeenth centuries. In: Continuity and Change 3 (1988), S. 177-208; Ole P. Grell u. a. (Hrsg.): Health Care and Poor Relief in Counter-Reformation Europe. London/New York 1999.

42 Ralf van Bühren: Die Werke der Barmherzigkeit in der Kunst des 12.-18. Jahrhunderts. Zum Wandel eines Bildmotivs vor dem Hintergrund neuzeitlicher Rhetorikrezeption. Hildesheim u. a. 1998, S. 282 und Abb. 122. - Von Carraccis angeblichen Sieben Werken der Barmherzigkeit, die in Holland (?) verkauft worden sein sollen, wie Gerard Hoet: Catalogus of naamlyst van schilderyen [...]. s'Gravenhage 1752, Bd. 1, S. 307, Nr. 1-7 berichtet (zit. bei Andor Pigler: Barockthemen. Budapest ${ }^{2} 1975$, Bd. 1, S. 540), ist sonst nichts überliefert.

43 Ein besonders schönes Beispiel einer 'Bettler-Caritas' ist wenig später auch das in mehreren Ausführungen überlieferte Gemälde des Bartolomeo Schedoni (Federica Dallasta, Cristina Cecchinelli: Bartolomeo Schedoni. Parma 1999, S. 158-161 [Kat. 61]); vgl. weiterhin L'allégorie dans la peinture. La représentation de la charité au XVII ${ }^{\circ}$ siècle. Caen 1986; Jutta Held: Caritas. Modelle einer Leidenschaft in den Bildkünsten der Frühen Neuzeit. In: Henry Keazor (Hrsg.): Psychische Energien bildender Kunst. Festschrift Klaus Herding. Köln 2002, S. 89-115; zur Vorgeschichte des 14. und 15. Jahrhunderts Ellen Schiferl: Caritas and the Iconography of Italian Confraternity Art. In: Studies in Iconology 14 (1995), S. 207-246. 
Betrachter gerade durch ihre prominent-isolierte Position verdeutlicht sie ist deshalb kompositionell isoliert und herausgehoben, um sie innerhalb der historia als Bildchiffre der theologischen Schlüsseltugend erkennbar zu machen. Das Wesen dieser Caritas wird zudem durch das aus der Rhetorik bekannte Verfahren des contrapposto, der kontrastierenden Gegenüberstellung, noch unterstrichen ${ }^{44}$ : Unmittelbar neben der Mutter erscheint im Hintergrund eine runzlige Alte, die zornig auf einen herandrängenden Vater mit Kind auf den Schultern zurückblickt aus Furcht, er könnte ihr das Almosen streitig machen. Indem die Alte so an Personifikationen des Geizes erinnert, wird ein maximaler Kontrast zur sich selbst veräußernden Nächstenliebe aufgemacht.

Erst einmal auf dieses Verfahren der Überlagerung von Sinn-Ebenen durch Herausstellung etablierter Bildchiffren aufmerksam geworden, erschließen sich mit entsprechend vorgeprägten Wahrnehmungsweisen auch die anderen Figurengruppen: Die Verbindung von blindem Musiker und jugendlichem Führer ist eine geläufige Erscheinung der Jahre um 1600 , wobei Blinden, die keine einfache Drehleiher, sondern ein Streichinstrument beherrschten, eine besondere Würde zugesprochen wurde es handelt sich hier kaum um ein Bild des ‘törichten Blinden', eher um einen Kollegen des blinden Homer und dessen Führer, dem Ziegenhirten Glaukos. Oder aber und naheliegender: Man erinnert sich des einzigen Blinden im Neuen Testament, der allein aufgrund seines bedingungslosen Glaubens an Christus, der ihn zum Messias eilen ließ, sehend wurde: "caeci in fidelitate Christi lumen aspiciant" - Blinde werden durch den Glauben an Christus sehend, so faßt es etwa der Kirchenvater Hieronymus zusammen; es ließen sich Hunderte weiterer Textbelege dafür anführen. ${ }^{45}$ Annibales Blinder ließe sich daher leicht als Hinweis auf den Glauben, der sehend machen kann, verstehen.

David Summers: Contrapposto. Style and Meaning in Renaissance Art. In: Art Bulletin 59 (1977), S. 336-371; auch Bertrand Rougé: Oxymore et "contrapposto", maniérisme et baroque. Sur la figure et le mouvement entre rhétorique et arts visuels. In: Etudes epistémè 9 (2006), S. 99-129 (Themenheft: Baroque/s et maniérisme/s litteraires: tonner contre?).

45 Hieronymus: Comm. in Amos (C), Vers. 6. - Ein Überblick zum Thema Blindheit bei Moshe Barasch: Blindness. The History of a Mental Image in Western Thought. New York u. a. 2001. - Zu Annibales besonderem Interesse an Blinden s. Anna Ottani Cavina: Studies from life. Annibale Carracci's paintings of the blind. In: Emilian painting of the 16 th and 17 th centuries. A symposium. Bologna 1987, S. 89-99; 
Zur dritten Figurengruppe, dem Kranken in der Schubkarre: Dieser Mann wird innerhalb der Bildlogik der historia offenbar trotz seines prekären Zustandes herbeigefahren, um die Aufmerksamkeit des Almosengebers Rochus zu erwecken und seine eminente Bedürftigkeit vor $\mathrm{Au}$ gen zu führen. Für den Betrachter dürfte diese Szene zugleich als eine Art proleptischer Verweis auf die erst später offenbar werdenden Heilkräfte des Rochus dienen, der mit Gottes Hilfe die Pest kurieren und zu einem der Nothelfer in Krankheit aufsteigen wird: Rochus als Almosengeber würde auf Rochus als Heiler vorausdeuten, die materiellen Gaben auf die immateriellen - erster Hinweis auf einen für die weitere Argumentation zentralen Aspekt. Die emporgerichteten Augen des Kranken spiegeln jedenfalls eine Mischung aus verzweifeltem Schmerz und intensiver Hoffnung - eine Hoffnung auf die Hilfe des Heiligen, die sich offenbar aus dem Glauben nährt, hält der Kranke doch einen Rosenkranz in der Hand. Das Begriffspaar 'Krankheit und Hoffnung' war für den Betrachter auch aus anderen Gründen naheliegend, finden sich beide Begriffe doch ausgehend von der Bibel in der Patristik und bei allen späteren Theologen engstens miteinander verbunden: Bereits Paulus schreibt im Römerbrief (Röm 5,5): "Durch die Hoffnung seid ihr gesundet", und Augustinus formuliert daraus noch bündiger: "spes parit salutem", aus Hoffnung entsteht (seelische) Gesundheit - auch in diesem Fall ließen sich die Nachweise beinahe beliebig fortsetzen. ${ }^{46}$

Wer immer sich dieser omnipräsenten theologischen Deutungen angesichts einer Blinden- und Krankengruppe erinnerte, für den mußten die drei Figurengruppen auf Carraccis Gemälde gerade durch ihre augenfällige Vereinzelung und Herausstellung wie Bildchiffren nicht nur von Caritas, sondern auch von Spes und Fides erscheinen. Carraccis drei Figurengruppen von Bedürftigen würden sich im Sinne der Christusworte (Mt 25,40): "Was ihr dem geringsten eurer Brüder getan habt, das habt ihr mir getan" unter dem Gewand der Bettler als die drei theologischen Tugenden von Glaube, Liebe und Hoffnung erweisen. Am

dies.: Annibale Carracci's painting of the blind. An addition. In: Burlington Magazine 131 (1989), S. 27 f.; Alessandro Brogi. In: Annibale Carracci 2006 (s. Anm. 18), S. 220 f. (Kat. IV.19-20).

46 Aurelius Augustinus: Epistolae (Opera omnia 39). Hrsg. von Armand B. Caillau. Paris 1842, Nr. 78. - Zu den theologischen Implikationen von Krankheit und Seuche im späteren 16. Jahrhundert zusammenfassend Boeckl (s. Anm. 32). 
eindeutigsten und schnellsten wäre in der Muttergestalt die altüberlieferte Caritas-Formel zu erkennen, die begleitenden Gruppen würden sich daran anschließend weniger als festgeprägte visuelle Formeln denn auf katechetisch-bibelexegetischer Ebene als feststehende Sinnbilder für Glaube und Hoffnung zu erkennen geben. Die exemplarische historia des Heiligenlebens erfuhr so in ihrem verosimile durch das Vermeiden von expliziten Personifikationen keine Störung; dennoch kommentierte sich das Gemälde quasi selbst, erinnerte und verwies an die in der Handlung wirkenden, allgemeingültigen christlichen Tugenden (virtù in specie e genere, um mit Paleotti zu sprechen, oder aber: schemata virtutum mit Molanus).

Welche Bedeutung kommt in diesem Kontext schließlich der prominent plazierten und bislang übergangenen Gruppe von Bedürftigen im linken Bildvordergrund zu - außer das Spektrum der Almosenempfänger noch um Waisen und Pilger zu erweitern? Die Handlung scheint zunächst ganz in genrehafter Detailbeobachtung befangen: Der zentralen sitzenden Gestalt, dem Familienvater, zeigt einer seiner Sprößlinge stolz die Münze, die er eben erhalten hat, sein Brüderlein wird derweil auf der anderen Seite gehalten und vergnügt sich mit einem Apfel. Das Mädchen mit dem Aussteuer-Beutelchen und die Mutter sehen diesem Geschehen zu, während ein jüngerer Mann dahinter seine Geldstücke abzählt. Dargestellt werden so freilich nicht nur die verschiedenen Altersstufen vom Säugling über diverse Stadien des Kindheit und Jugend bis hin zum Erwachsenen. Vorgeführt werden insbesondere auch die alterstypischen Reaktionen auf Besitz: Der kleine Bub freut sich noch mehr an einem Apfel als an Geld, sein etwas älterer Bruder erahnt zwar die prinzipielle Bedeutung von Münzen, kann aber noch nicht den Wert von einer oder mehrer solcher Münzen unterscheiden, wogegen der junge Mann sein Almosen genau abzählt; aber auch die Pilgerin läßt ihre Geldstücke durch die Hände gleiten. Genau diese Konstellation aber - Kinder mit Äpfeln, Erwachsene mit Geld - dient seit der Patristik als mahnendes Beispiel für den richtigen Umgang mit Besitz, seinem relativen Wert, und als Warnung vor Habsucht. Das bei Katholiken wie Protestanten diskutierte Problem bringt Luther auf den Punkt: "Die lieben Kindlein leben in Unschuld [...], ohne Neid, Zorn, Geiz, [...] sie nehmen ei- 
nen Apfel für einen Groschen. Daher auch Christus uns Alten ernstlich anredet, ihrem Exempel nachzufolgen [...]." ${ }^{47}$

Annibales Gemälde vollzieht so einen Wechsel des Adressatenkreises: Stellt es mit dem Rochus den Bruderschaftsmitgliedern ein leuchtendes Exemplum christlicher Taten der Barmherzigkeit vor Augen, so richtet es sich mit der Gruppe von Bedürftigen an die Almosenempfänger und erinnert diese an den richtigen Umgang mit den Gaben. Wenn aber das Gemälde in gewisser Weise zweigeteilt seine Botschaft vorträgt, dann ließe sich zumindest überlegen, ob es Zufall ist, daß die genrehafte Vordergrundsgruppe in unmittelbarer, leicht faßlicher Evidenz Reaktionen auf Gaben vorführt, daß dagegen das Tun des Rochus im rechten Mittelgrund eine anspruchsvollere Wahrnehmungsleistung verlangt, nämlich in den drei herausgestellten Figurengruppen zugleich Sinnbilder der theologischen Tugenden und damit eine Art Kommentar und allgemeingültige theologische Explikation des Ereignisses zu erkennen. Wäre dem so, dann würde Annibale die vielleicht komplizierteste gegenreformatorische Anforderung an christliche Bilder erfüllen: In einer einzigen Darstellung ganz verschiedene Bevölkerungs- und Bildungsschichten gleichermaßen anzusprechen und deren unterschiedliche Erwartungs- und Verstehenshorizonte einzulösen - ein (allerdings auch schon für die Zeitgenossen nicht ganz einfach zu konzeptualisierendes) Ideal der Bilder, das insbesondere der Bologneser Erzbischof Gabriele Paleotti als "consenso universale del popolo" gepriesen hatte. ${ }^{48}$

\section{Carità und grazia, novità und natura}

Es dürfte kein Zufall sein, daß Annibale gerade an einem historischen Exemplum der Almosenvergabe seine neue Bildsprache entwickelte und die drei theologischen Tugenden als visuellen Subtext und Kommentar

47 Martin Luther: Werke. Kritische Gesamtausgabe. Tischreden, Bd. 1. Weimar 1912, S. 311, Nr. 660; das Beispiel ließ sich aber auch im Sinne kindlicher Habsucht und damit der Erbsünde deuten. So Aurelius Augustinus: Confessiones. Hrsg. von Joseph Bernhart. Darmstadt 1984, 2, 4, und wieder Luther: Werke. Kritische Gesamtausgabe. Tischreden, Bd. 2. Weimar 1913, S. 124, Nr. 1532.

48 Paleotti (s. Anm. 40), S. 497, vgl. auch S. 496 zur "grazia universale". - Am ausführlichsten kommentierend dazu Ulrich Heinen: Rubens zwischen Predigt und Kunst. Der Hochaltar für die Walburgenkirche in Antwerpen. Weimar 1996, S. 39-44. 
aufrief. Als Anlaß für die Invention läßt sich ein, wenn nicht der zentrale Streitpunkt zwischen Reformierten und Altgläubigen vermuten: Gemeint sind die Themen von Gnade, Jüngstem Gericht, Rechtfertigung und Werkgerechtigkeit des Menschen. Welchen Anteil kann der Mensch an seiner Erlösung nehmen, allein gläubig auf Gottes Gnade zu vertrauen oder aber in einer Art 'heiligem Tauschhandel' - sacrum commericum ist der zeitgenössische Begriff dafür -, durch gute Taten und Gaben also, aktiv an seiner Erlösung mitwirken bzw. von anderen für sich mitwirken lassen? Die katholischen Theologen des 16. Jahrhunderts und insbesondere die Trienter Konzilsbeschlüsse bekräftigten gegen die reformatorische Kritik unablässig den Wert der über die traditionellen Christenpflichten hinausgehenden Sonderleistungen der Caritas in Form von Almosen und verwandten Formen der Misericordia für das Seelenheil des Stifters. ${ }^{49}$ Zwar war, so wurde betont, damit allein noch keine Erlösung zu 'erkaufen', aber in Verbindung mit dem festen Glauben durfte der Stifter auf einen bevorzugten Gnadenerweis Gottes hoffen. Annibales Gemälde scheint daher insbesondere diesen Zusammenhang von karitativer Gabe, Glaube und Hoffnung auf Gnade herauszustellen: Den Mitgliedern der Rochus-Bruderschaft führte das Gemälde die Legitimität und den Wert ihres mildtätigen Engagements vor Augen und betätigte und ermunterte sie zugleich dazu.

Betont wird in Annibales Darstellung der Schritt von der materiellen Gabe zur geistig-seelischen Tugend und damit letztlich zur Erlösung. Eine zumindest verwandte Denkbewegung expliziert auch der Jesuit Julius Roscius (Giulio Rossi da Orte) in seinem 1586 in Rom publizierten und reich illustrierten Traktat zu den Werken der Barmherzigkeit mit dem Titel Icones operum misericordiae. ${ }^{50}$ Allein schon die Titelblätter

49 Das Konzil von Trient hielt in Sess. VI, can. 32 explizit fest: "Si quis dixerit, hominis iustificati bona opera ita esse dona Dei, ut non sint etiam bona ipsius iustificati merita, aut ipsum iustificatum bonis operibus, quae ab eo per Dei gratiam et Iesu Christi meritum (cuius vivum membrum est) fiunt, non vere mereri augmentum gratiae, vitam aeternam et ipsius vitae aeternae (si tamen in gratia decesserit) consecutionem, atque etiam gloriae augmentum: anathema sit." (Zit. nach Heinrich Denzinger: Enchiridion Symbolorum. Freiburg i. Br. ${ }^{28} 1952$, S. 299).

so Roscius (s. Anm. 37). Die kunsthistorische Bedeutung dieses Traktates erkannte Christine Göttler: Die Kunst des Fegefeuers nach der Reformation. Kirchliche Schenkungen, Ablaß und Almosen in Antwerpen und Bologna um 1600. Mainz 1996, S. 23-36; offenbar bislang nicht benannt ist dagegen das wichtigste Vorbild für 
zum ersten und zweiten Teil lassen deren gesamte Grundstruktur erkennen (s. Abb. 9, 10): Zunächst werden die bekannten Sieben Werke materieller Barmherzigkeit vorgestellt, danach die Sieben Werke geistiger Barmherzigkeit, also etwa die Unwissenden zu belehren, die Traurigen zu trösten oder aber die Sünder auf den richtigen Weg zurückzuführen. Auf diese geistige Hilfestellung soll die Nächstenliebe in letzter Konsequenz hinzielen - denn natürlich genügt es nicht, nur körperliche Bedürfnisse zu befriedigen, wenn es weiterhin an der Seele krankt. ${ }^{51}$ Ganz entsprechend erinnert Annibales zunächst die materielle Gabe thematisierendes Rochus-Gemälde auf einer zweiten Ebene an die theologischen Tugenden als den eigentlichen Referenzpunkten des Almosengebens. Die Bedeutung dieser Ideen für das Gemälde reicht aber noch weiter: Roscius erläutert auch, daß für die Reichen und Vornehmen die Sorge um das materielle Kirchengebäude und seine Ausstattung eine besondere Form des Almosengebens und damit sowohl der Gottes- als auch der Nächstenliebe an der Gemeinschaft darstelle - ein Gedanke, der, wenn nicht seinen Ursprung, so doch einen wichtigen Impuls den Lobschriften auf das Pontifikat Gregors XIII. (1572-1585) und dessen Stiftungen verdankte. ${ }^{52}$ Auf Annibales Gemälde übertragen, ergibt sich, daß dieses nicht nur qua Bildinhalt der Rochus-Bruderschaft die Bedeutung von materiellen Almosen vor Augen führte, sondern daß das Stiften eben dieses Gemäldes selbst bereits einen solchen Akt des Almosen-Gebens

die von Mario Cartaro ausgeführten Stiche in Roscius: die Septem opera misericordiae corporalia des Philips Galle von 1577, diese etwa bei van Bühren (s. Anm. 42), S. 293-297 (Nr. 52). - Vgl. für die Praxis und Rom etwa Reginald Grégoire: "Servizio dell'anima quanto del corpo" nell'ospedale romano di Santo Spirito (1623). In: Richerche per la storia religiosa di Roma 3 (1979), S. 239-241.

51 Roscius (s. Anm. 37), S. 48 f.: "Quid prodest cibus, aut potus, aut vestis, aut libertas, aut sanitas, aut a caeli iniurijs defendens textum, aut extrema decendentium domus sepulchrum, si peccato perit anima?" - Die Gesamtaussage des Traktates wird auch noch einmal durch drei Diagramme - Tabula I. de distinctione VII. operum Misericordiae corporalium et spiritualium [...] usw. - nach Thomas von Aquin und Alexander von Hales zusammengefaßt.

52 Ebd., fol. br: "Est igitur illud etiam eleemosynae genus Deo pergratum, quae in divini cultus ornatum extructionem, ac reparationem ecclesiarum refertur. Cuius operis usus praecipue a magnatibus atque opulentis viris postulatur, qui uno tempore \& Deum honorant, \& non obscura populis tenuioribus eleemosynam elargiuntur, quibus commoditatem praebent, ut patefactis templis rei divinae, suarumque animarum saluti vacare possint." - Dazu ausführlich Göttler (s. Anm. 50), S. 42-56. 


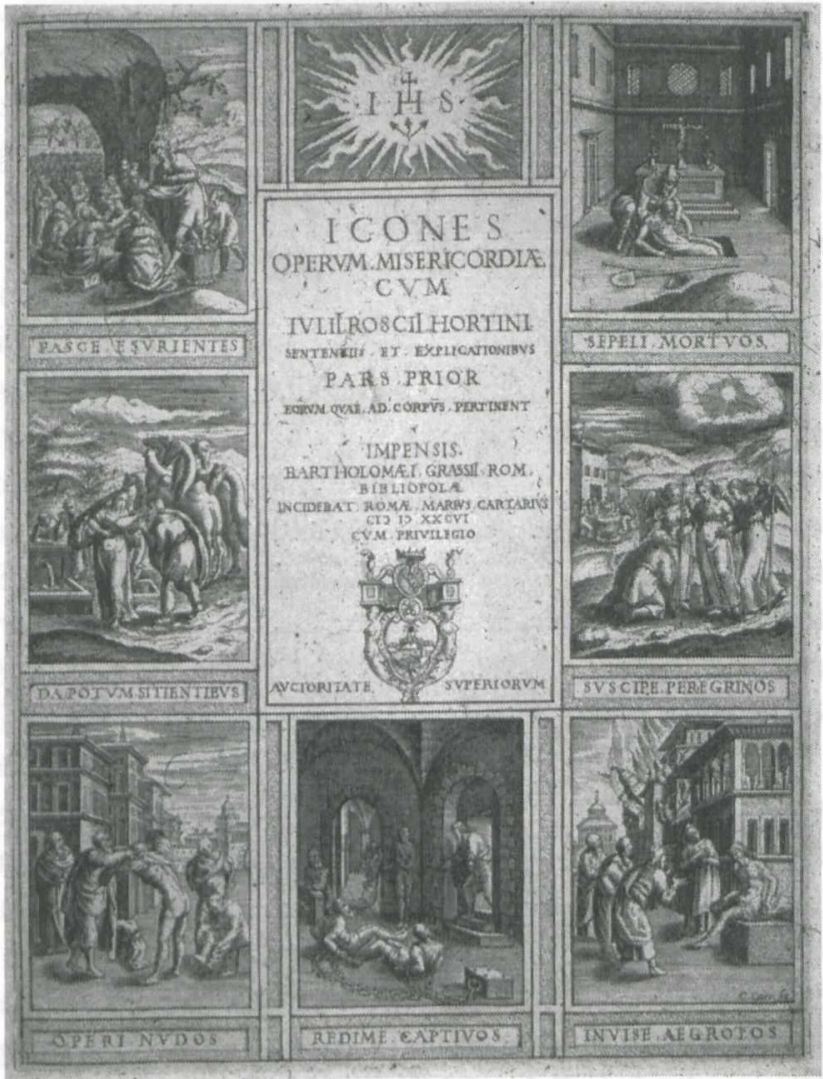

Abb. 8: Julius Roscius Hortinus: Icones operum misericordiae. Rom 1586, Titelblatt des ersten Teils. 
darstellte - und zwar einen besonders wirkmächtigen Akt, versinnbildlicht die Almosenspende des Hl. Rochus doch just den geistigen Sinn und das Ziel von guten Taten für Geber und Empfänger gleichermaßen. Die materielle Gabe des Kunstwerks will nicht nur die Kirche schmücken, sondern v. a. auch als geistige Gabe die Betrachter belehren, zum karitativen Tun ermuntern, Hoffnung und Trost für die Bedürftigen spenden usw. - exakt die von Roscius entwickelten Stichworte der geistigen Werke der Barmherzigkeit.

Indem schließlich der Künstler, Annibale Carracci, für dieses Gemälde und zu diesem Zweck eine neue Bildsprache erfindet, leistet auch er einen über das Normalmaß der Christenpflicht weit hinausgehenden Beitrag geistiger Barmherzigkeit und Nächstenliebe. Zu überlegen ist, ob Annibale sein Gemälde als eine Form malerischer Misericordia und Caritas verstand. Daß er in seinem Brief an die Bruderschaft verspricht, den Auftrag auf jeden Fall für den "himmlischen Fürsprecher, S. Rocco" fertigzustellen, scheint noch nicht über mehr oder weniger formelhafte Standardaussagen hinauszugehen, vergleicht man etwa die Wendung Tintorettos, der wenig früher, 1577, den Mitgliedern der Scuola Grande di S. Rocco in Venedig schreibt, er würde für sie praktisch kostenlos "aus Verehrung für den Heiligen" arbeiten. ${ }^{53}$ Ungewöhnlicher erscheint dagegen die Antwort der Bruderschaft an Annibale, die ihm wünscht: "Möge Gott der Herr auf die Bitten des Hl. Rochus hin Euch und Eurem Pinsel die Gnade und Kraft verleihen, in vollkommenem Wissen (bzw. in vollkommener Wissenschaft - "perfetta scienza") die Historien Gottes und seiner Heiligen und aller anderer darzustellen". ${ }^{54}$ Annibales vollendetes Wissen als christlicher Maler wird hier als Resultat eines Gnadenaktes Gottes verstanden, eine Gnade oder grazia, die ihrerseits als Antwort auf Annibales Glaube, Hoffnung und Caritas zu begreifen ist. ${ }^{55}$

53 Astrid Zenkert: Tintoretto in der Scuola di San Rocco. Tübingen 2003, S. 21 f.

54 Giovanna Perini: Gli scritti dei Carracci: Ludovico, Annibale, Agostino, Antonio, Giovanni Antonio. Bologna 1990, S. 155-157 (Nr. 4 f.). - Vgl. den späteren Fall eines Auftrags an Guido Reni, eine Tafel mit dem Triumph des Hiob für die Arte della Seta in Bologna zu malen, in dem davon die Rede ist, Gott möge dem Maler "gratia” verleihen; dazu Gabriele Wimböck: Guido Reni (1575-1642). Funktion und Wirkung des religiösen Bildes. Regensburg 2002, S. 38-42.

55 Zum Konzept von grazia vgl. Patricia Emison: Grazia. In: Renaissance Studies 5 (1991), S. 427-460; Richard E. Spear: The 'Divine' Guido. Religion, Sex, Money and Art in the World of Guido Reni. New Haven/London 1997, S. 102-127. 


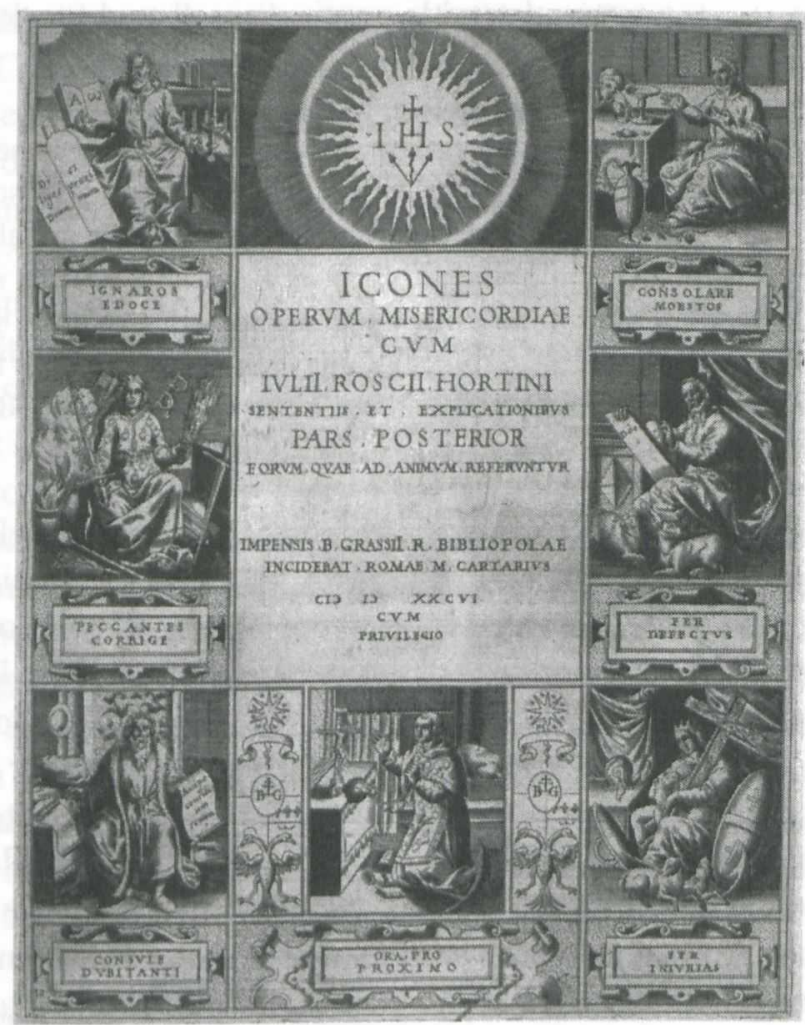

Abb. 9: Julius Roscius Hortinus: Icones operum misericordiae. Rom 1586, Titelblatt des zweiten Teils. 
Auch in dieser Hinsicht eröffnen sich enge Bezüge zu Paleottis idealem pictor christianus, von dem es heißt: "Il fine principale serà, col mezzo della fatica et arte sua acquistarsi la grazia divina", und für dessen 'karitative Malerei' gilt:

ella fa e rappresentare dinanzi agli occhi persone cumulatissime de meriti e che per la loro essemplare vita, piena d'ogni virtù, sono state sopra modo grate a Dio. [...] essendo tutte le azioni proprie di quella virtù, al fine della quale sono ordinate, non avendo altra mira in somma tutte le sacre imagini, mediante gli atti religiosi che rappresentano, che di unire gli uomini con Dio, che è il fine della carità: ne segue manifestamente che l'essercitio del formare imagini si ridurrà alla carità, e perciò diverrà virtù degnissima e nobilissima. ${ }^{56}$

Vor dem Hintergrund von Roberto Zapperis wichtiger Klarstellung, daß Paleotti und die Carracci aller Wahrscheinlichkeit nach keinen Austausch pflegten, ja im Gegenteil Paleotti die Künstlergeneration vor den Carracci zu favorisieren schien, lassen sich die festgestellten Parallelen zum Zusammenhang von künstlerischer carità und grazia jedoch nur so verstehen, daß Theologe und Maler unabhängig voneinander den Konsens einer intensiv und weithin geführten Diskussion festhielten. ${ }^{57}$

So große Gemeinsamkeiten dieser zeitgenössische Kunstdiskurs offenbar zwischen Annibale Carraccis visuellen und Gabriele Paleottis schriftlichen Äußerungen auch stiftete, in einem Punkt scheinen beider Meinungen grundlegend zu differieren: nämlich in Bezug auf künstlerische novità. Der Kardinal formulierte - allen Beteuerungen, "die Hände der Maler nicht binden zu wollen” zum Trotz - eine eher traditionsbewußt-konservative Position. ${ }^{58}$ Carracci dagegen zielt seit Beginn seiner Karriere auf eine Erneuerung der Malkunst und speziell mit seinem $\mathrm{Hl}$. Rochus auf eine neue, göttlich inspirierte Bildsprache christlicher historia im Geiste von Trient - zumindest betont dies mein Beitrag von Anbeginn an. Aber worin genau besteht diese 'Neuheit'? Denn

56 Paleotti (s. Anm. 40), S. 210 und S. 161 f., vgl. auch S. 215. - Vgl. das kurze Resüme bei Luise Leinweber: Bologna nach dem Tridentinum. Private Stiftungen und Kunstaufträge im Kontext der katholischen Konfessionalisierung. Das Beispiel San Giacomo Maggiore. Hildesheim u. a. 2000, S. 219 f.

57 Zapperi (s. Anm. 14).

58 Paleotti (s. Anm. 40), S. 398-407, das Zitat S. 406. 
Annibales Grundprinzip einer doppelten Lese-Ebene ist alles andere als neu: So arbeitet etwa Jacopo Bellini mit einer ähnlichen Verbindung von Erzählung und Kommentierung bei seiner Zeichnung einer Kreuztragung Christi: Der stürzende römische Reiter läßt sich auf einer zweiten Verstehensebene als Sinnbild der superbia verstehen und damit als Hinweis darauf, daß entgegen dem Anschein mit dem Tod Christi der Niedergang des vermeintlich siegreichen römischen Reiches unabwendbar wird. ${ }^{59}$ Und Raffael hat seinen Borgobrand so angelegt, daß die Figurengruppen des Vordergrundes nicht nur im Sinne der Erzählstruktur einer aristotelischen Tragödie zu verstehen sind, sondern auch als allgemeine Sinnbilder etwa der Fürsorge und pietas erga parentes - allein dann läßt sich z. B. die prominente Äneas-Anchises-Gruppe links erklären, die vom eigentlichen Thema nicht vorgegeben ist, jedoch durch den Rekurs auf die bekannte Bildchiffre Affekte, Tugenden und Vorbild des Troja-Brandes aufruft. ${ }^{60}$ Wenn schließlich eine zwar erst um die Mitte des 17. Jahrhunderts von Giovanni Svizzero gestochene, von Matteo Bolzetta herausgegebene Serie der Sieben Werke der Barmherzigkeit tatsächlich ein verlorenes Vorbild des Cinquecento reproduziert, dann wäre dort sogar schon eine Mutter-Kind-Gruppe auf der ersten Darstellung und quasi am 'Eingang' der Folge als 'versteckt-kommentierende' Caritas-Figur zu sehen (s. Abb. 11). ${ }^{61}$ Im 17. Jahrhundert findet sich dieses Prinzip dann aufgegriffen etwa bei Rubens oder Guido Reni. ${ }^{62}$

59 Felix Thürlemann: Geschichtsdarstellung als Geschichtsdeutung. Eine Analyse der Kreuztragung (Fol. 19) aus dem Pariser Zeichnungsband des Jacopo Bellini. In: Wolfgang Kemp (Hrsg.): Der Text des Bildes. Möglichkeiten und Mittel eigenständiger Bilderzählung. München 1989, S. 89-108.

60 Kurt Badt: Raphael's 'Incendio del Borgo'. In: Journal of the Warburg and Courtauld Institutes 22 (1959), S. 35-59; Rudolf Preimesberger: Tragische Motive in Raffaels “Transfiguration”. In: Zeitschrift für Kunstgeschichte 50 (1987), S. 89-115.

61 Frank Jewett Mather Jr.: A Titan Problem. The Seven Acts of Mercy. In: Gazette des Beaux-Arts 84/22 (1942), S. 165-172; van Bühren (s. Anm. 42), S. 276 (Nr. 22); vgl. hier auch ein Blatt aus der Serie Lob der Frauentugenden des Dirck Volckertsz. Coornhert von 1555. - Ein weiteres mögliches Beispiel in Prospero Fontanas Bologneser Almosenspende des Hl. Alexis von 1574/76 analysiert Leinweber (s. Anm. 56), S. 159-166.

62 Ohne daß hier die Frage der Vermittlung, Abhängigkeit oder aber annähernd parallelen, eigenständigen Erfindung geklärt werden könnte, s. Heinen (s. Anm. 48), S. 57 und Wimböck (s. Anm. 54), S. 182-192. 
Entscheidend ist bei alledem freilich nicht, daß Carracci ein wirklich vollkommen neues Prinzip christlicher Bilderzählung erfunden hat, sondern daß er diese Lösung bei der Almosenvergabe des $\mathrm{Hl}$. Rochus mit neuer Konsequenz und emphatisch als seinen Vorschlag inszenierte. Dies war ihm freilich nur vor dem andersartigen Hintergrund seiner früheren radikalen Naturnachahmung und 'vorbildlosen Neubegründung' der Kunst möglich: Indem Annibale in seinen frühen Jahren vorgeblich auf jegliche Ausbildung in der künstlerischen Tradition verzichtet, seine Malweise weitgehend als naturbegabter Autodidakt und ausschließlich am Vorbild der Natur orientiert erlernt hatte, konnte er sich (im Rahmen dieses Vorstellungsmodells) der übermächtigen Kunst-Tradition entziehen und etwas 'Neues' begründen. So jedenfalls lautete die Argumentation zu novità, die in ihren Grundbestandteilen zwar wiederum bezeichnenderweise auf antike Künstlermythen zurückgeht, aber von der Mitte des Cinquecento an ungewohnte Bedeutung und Betonung gewinnt: Vielleicht am prominentesten wird in Juan Huartes wegweisender Abhandlung über die verschiedenen menschlichen Begabungen von 1575 (italienische Übersetzung 1582) für die höchste Geistes-Stufe festgeschrieben, daß diese ohne Lehrmeister und nur am Vorbild der Natur orientiert Wunderbares zu leisten vermöge. ${ }^{63}$ Dagegen scheinen Vorstellungen, die aus heutiger Sicht viele eher beim Gedanke an grundstürzende Innovation in den Sinn kommen und die auch schon in der Frühen Neuzeit eine zentrale Rolle spielten: Inspiration und Schöpfertum, gerade für unsere Frage nach novità nicht federführend beizutragen. ${ }^{64}$

63 Juan Huarte: Essame de gl'Ingegni de gli Huomini. Venedig 1582, S. 73 f., wo es zur zweiten und dritten 'Begabungsstufe' heißt: "Altri ingegni s'alzano un grado più sù: perche sono piacevoli, \& facili nell'apprendere le cose [...]. Di questi si può verificare quella tanto celebrata sentenza d'Aristotele: Il nostro intelletto è come una tavola piollata, nella quale neßuna cosa è dipinta. Perche tutto quello, che hanno da sapere, \& apprendere, bisogna che prima l'odano da un'altro, \& sopra ciò non hanno inventione alcuna. Nel terzo grado fa la natura alcuni ingegni tanto perfetti, che non hanno bisogno di maestri, i quali insegnino loro [...]." - Vgl. ausführlicher zu diesen Zusammenhängen Pfisterer (s. Anm. 14).

64 Vgl. zu diesen Fragen jüngst Christoph J. Steppich: Numine afflatur. Die Inspiration des Dichters im Denken der Renaissance. Wiesbaden 2002; Patricia A. Emison: Creating the 'divine' artist. From Dante to Michelangelo. Leiden 2004; Maria Ruvoldt: The Italian Renaissance imagery of inspiration. Metaphors of sex, sleep, and dreams. Cambridge u. a. 2004. 


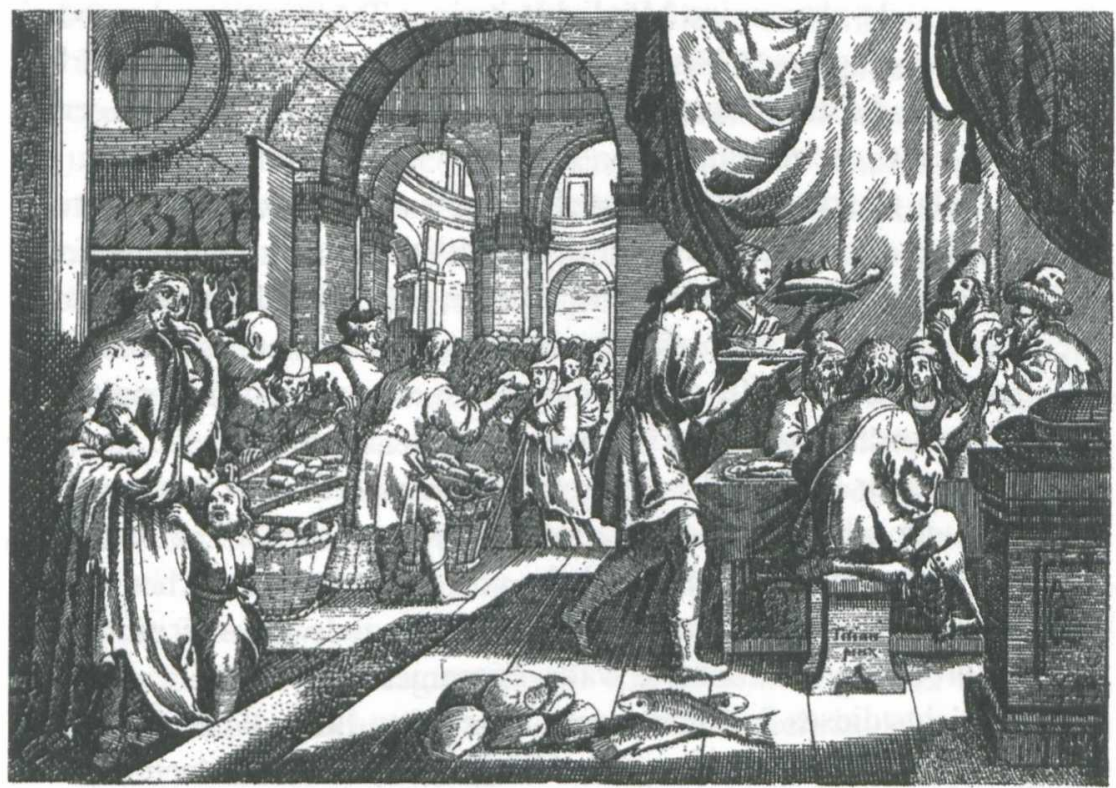

Suscipe, pasce, ciba, si se.tibi sisfat. egenus.

Clibore de Vita crena parata tibi. in Padowa p.M. Molketta

Abb. 11: Giovanni Svizzero: Armenspeisung aus einer Serie der Sieben Werke der Barmherzigkeit, Privatsammlung. 
Als entscheidend für die Jahrzehnte vor 1600 erweist sich jedenfalls: Radikale, autodidaktische Naturnachahmung gilt als eine der wichtigsten, wenn nicht als einzige Möglichkeit eines Traditionsbruchs und einer voraussetzungslosen Neubegründung der Kunst! Allein das Vorbild der Natur und die angeborene Begabung ermöglichten es, einen eigenen, neuen Weg einzuschlagen.

Annibale Carracci verstand sich als ein solcher radikaler Autodidakt - wobei dies natürlich nicht der Wirklichkeit entsprach, aber erstmals stilisierte sich ein Künstler von seinen ersten Werken an so konsequent nach dieser Vorgabe, daß ihm selbst noch die moderne Forschung in Teilen folgt: So schaffte es der Maler etwa, weitgehend zu verwischen, bei wem er tatsächlich in die Lehre gegangen war; statt dessen forcierte er die Idee einer (mehr oder weniger autodidaktischen) 'Carracci-Akademie', in der allein nach der Natur gearbeitet wurde. Ganz in diesem Sinne werden alle seine frühen Werke von den zeitgenössischen Kunstkritikern als radikale Naturnachahmung ohne jede künstlerische oder kunsttheoretische Überhöhung wahrgenommen. ${ }^{65}$

Angesichts dieses Selbstentwurfs, angesichts dieser Wahrnehmungsfolie markiert die Almosenvergabe des $\mathrm{Hl}$. Rochus den entscheidenden Wendepunkt in der Bildsprache des Künstlers, an diesem Werk wird wohl erstmals in aller Deutlichkeit der überraschend schnelle Wandel von Annibale Carracci, dem radikalen Naturnachahmer, zu Annibale Carracci, dem Meister idealisierend-monumentaler Naturdarstellung, deutlich, bei dem die künstlerische Tradition nun doch wieder eine zentrale, wenngleich neuartige Rolle als versatzstückartiges Formelement und als Bedeutungsträger zu spielen beginnt: Deutlich wird dies, um nur noch ein Beispiel anzuführen, an der Caritas-Gestalt, die sehr genau eine Figur

65 Aus dieser kunstvollen Verstellung, die mit großem intellektuellem und theoretischem Aufwand auf 'Kunstlosigkeit' zielte, erklärt sich meiner Auffassung nach letztlich auch der Forschungsstreit um die kunsttheoretische Bildung der Carracci; vgl. zu den verschiedenen Positionen zusammenfassend Charles Dempsey: Annibale Carracci and the beginnings of baroque style. Fiesole ${ }^{2} 2000$; Carl Goldstein: Visual Fact over Verbal Fiction. A Study of the Carracci and the Criticism, Theory, and Practice of Art in Renaissance and Baroque Italy. Cambridge u. a. 1988; zu den gesichert eigenen Äußerungen der Carracci vgl. Henry Keazor: Distruggere la maniera? Die Carracci-Postille. Freiburg i. Br. 2002. - Zum Kontext der Carracci-Akademie immer noch wichtig David Rosand: The Crisis of the Venetian Renaissance Tradition. In: L'Arte (1970) 11/12, S. 5-53. 
aus Baldassare Peruzzis berühmtem Fresko des Tempelgang Mariens in S. Maria dell'Anima zu Rom zitiert; von dort leitet sich ihre hochragende und fest modellierte Monumentalität her, die so auffällig mit den anderen Bildfiguren Carraccis kontrastiert. Dieser Unterschied scheint insbesondere auch durch die Gruppe von Krankem und Schubkarren-Fahrer unmittelbar daneben betont, in denen besonders deutlich die für die Carracci-Akademie charakteristischen Natur- und Aktstudien in kompliziert verkürzter Ansicht eingesetzt sind. ${ }^{66}$ Es scheint fast, als solle die wichtigste theologische Tugend, die ja nicht nur den irdischen amor proximi, sondern auch den amor Dei und damit einen göttlichen Anteil umfaßt, durch den Rekurs auf die ideale Hochrenaissance-Malerei in einer weiteren Verstehensschicht ausgezeichnet und überhöht werden.

Annibales novità in der Almosenspende des $\mathrm{Hl}$. Rochus erweist sich so als neues, reflektiertes Verhältnis von 'unmittelbarer Naturschilderung' und 'interpikturalem' Einsatz des künstlerischen Erbes: Annibale diente die Tradition - anders als bei früheren Künstlern - nicht mehr selbstverständlich als Ausgangspunkt von Bildfindungen. Er nutzt Vorbilder und Bild-Gedächtnis erst in einem zweiten Schritt dazu, komplexe Erzähl- bzw. Darstellungs-Möglichkeiten und Sinn-Aufladungen zu gewinnen. Dabei ermöglicht ihm der freie Umgang mit den älteren Bildsprachen, für einzelne Elemente seiner Gemälde jeweils das am besten Angemessene aus dieser Tradition aufzugreifen und zusammenzuführen. Es ist diese Synthese-Leistung des jeweils Besten, die die Kunstliteratur des 17. Jahrhunderts (und neuerdings auch wieder die moderne Forschung) an Annibale besonders lobt - ein Vorgehen, das ansatzweise auch schon im Cinquecento und speziell zu Beginn von Annibales Karriere, 1581, von Lomazzo in seinem Malereitraktat als zukünftiges Ideal der Kunst entwickelt wurde: Die Farbe Tizians, den Disegno Michelangelos, die Weichheit Correggios usw. - kurz: das Beste aus allen italienischen Malerschulen - am jeweils richtigen Ort eines einzigen $\mathrm{Ge}$ mäldes zu vereinen. ${ }^{67}$ Möglich wird dieser neuartige, hochbewußte und

Zur Vorzeichnung für den Schubkarren-Fahrer in Budapest zuletzt Catherine Loisel Legrand. In: Daniele Benati u. a. (Hrsg.): The Drawings of Annibale Carracci. Washington 1999, S. 106 f. (Kat. 26) und Alessandro Brogi. In: Annibale Carracci (s. Anm. 18), S. 270 f. (Kat. V.18).

67 Dazu etwa Martin Kemp: 'Equal excellences'. Lomazzo and the explanation of individual style in the visual arts. In: Renaissance Studies 1 (1987), S. 1-26; Robert 
bedeutungshaltige Einsatz der künstlerischen Tradition für Carracci aber eben nur vor dem Hintergrund seines 'radikalen Traditionsbruches' und seiner 'reinen Naturnachahmung' im Frühwerk. Erst nachdem sich Annibale von allen Abhängigkeiten befreit und erst als diese seine Neubegründung der Malerei allgemeines Ansehen gefunden hatte, im Moment großer auswärtiger Aufträge also, erst in diesem Moment konnte er damit beginnen, die künstlerische Tradition in neuer Bewußtheit als 'Bedeutungsträger' wieder in seine Werke einzubauen und so quasi das künstlerische Potential von Anfang und Ende zusammen zu führen: Diesen Moment markiert die Almosenspende des Hl. Rochus. Der 'moderne' Carracci hätte damit erreicht, was die Antike für unmöglich gehalten hatte $^{68}$ : Nämlich den Neuanfang einer Kunst und deren Vollendung in einer Person zu vereinen.

Williams: Art, Theory and Culture in Sixteenth-Century Italy. Cambridge u. a. 1997, v. a. S. 123-186; Victor I. Stoichita: Ars ultima. Bemerkungen zur Kunsttheorie des Manierismus. In: Anne-Marie Bonnet, Gabriele Kopp-Schmidt (Hrsg.): Kunst ohne Geschichte? Ansichten zu Kunst und Kunstgeschichte heute. München 1995, S. $154-162$.

68 Etwa Quintilian: Institutiones Oratoriae, 10, 2; vgl. auch Quint (s. Anm. 3). 